This is a postprint version of the following published document:

Santos T. dos, et al. Modeling dynamic spherical cavity expansion in elasto-viscoplastic media, In: Acta Mechanica, 231(6), June 2020, Pp. 2381-2397

DOI: https://doi.org/10.1007/s00707-020-02646-2

(C) Springer-Verlag GmbH Austria, part of Springer Nature 2020 


\title{
Modeling dynamic spherical cavity expansion in elasto-viscoplastic media
}

\author{
T. dos Santos ${ }^{\mathrm{a}}$, A. Brezolin ${ }^{\mathrm{b}}$, R. Rossi ${ }^{\mathrm{b}}$, J. A. Rodríguez-Martínez ${ }^{\mathrm{c}, *}$ \\ ${ }^{a}$ Departamento de Engenharia Mecânica, Universidade Federal de Santa Maria, Av. Roraima, 1000, Prédio \%, Santa Maria, RS, \\ 97105-900, Brazil. \\ ${ }^{b}$ Departamento de Engenharia Mecânica, Universidade Federal do Rio Grande do Sul, R. Sarmento Leite, 425, Porto Alegre, RS, \\ Brazil. \\ ${ }^{c}$ Department of Continuum Mechanics and Structural Analysis, University Carlos III of Madrid, Avda. de la Universidad, 30, 28911 \\ Leganés, Madrid, Spain
}

\begin{abstract}
In this paper, we extend the dynamic spherical cavity expansion model for rate-independent materials developed in refs. $[1,2,3]$ to viscoplastic media. For that purpose, we describe the material behavior with an isotropic Perzynatype overstress formulation $[4,5]$ in which the material rate-dependence is controlled by the viscosity parameter $\eta$. The theoretical predictions of the cavity expansion model, which assumes that the cavity expands at constant velocity, are compared with finite element simulations performed in ABAQUS/Explicit [6]. The agreement between theory and numerical simulations is excellent for the whole range of cavitation velocities investigated, and for different values of the parameter $\eta$. We show that, as opposed to the steady-state self-similar solutions obtained for rate-independent materials $[1,2,3]$, the material viscosity leads to time-dependent cavitation fields and stress relaxation as the cavity enlarges. In addition, we also show that the material viscosity facilitates to model the shock waves that emerge at the highest cavitation velocities investigated, controlling the amplitude and the width of the shock front.
\end{abstract}

Keywords:

Dynamic cavity expansion, Shock waves, Viscoplasticity, Stress relaxation

\section{Introduction}

Beginning with the pioneering works of Hopkins [7] and Goodier [8], dynamic cavity expansion theories have been widely applied to model high-velocity penetration of metallic targets. The cavity expansion models use the solution of the pressure for expansion of a cavity in an infinite medium as the contact pressure experienced by the projectile during the penetration process. Over the years, several works have shown that penetration models based on cavity expansion theories provide fair predictions for the resisting force, ballistic limit and penetration depth $[9,10,1,11,12,13]$

Most of the cavity expansion theories assume that the elastoplastic fields that develop near the cavity are steadystate and self-similar $[10,14,15,1]$, such that the solution of the problem depends on only one space variable, and it

${ }^{*}$ Corresponding author. E-mail address: jarmarti@ing.uc3m.es 
is independent of time. Steady-state self-similar approaches have been applied to model penetration of targets which exhibit a range of material response including compressibility, anisotropy, strain hardening, pressure sensitivity and porosity $[10,1,13,16,17,18]$. However, since viscosity leads to time-dependent cavitation fields [15, 19, 20], steadystate similarity solutions are not possible for rate-dependent materials, imposing a limitation on the application of cavity expansion theories to model the penetration of targets with strain-rate dependent response. An important exception is the work of Warren and Forrestal [19], in which the cavity expansion model derived by Hopkins [7] was extended to consider the strain-hardening and strain-rate effects on the penetration of 6061-T651 aluminum targets impacted by spherical-nosed rods. Warren and Forrestal [19] showed that, for strain-rate sensitive materials, the cavitation fields depend on the current cavity radius. Moreover, the problem of dynamic expansion of pressurized cavities in viscoplastic compressible solids, with application to geological materials, was addressed by Cleja-Tigoiu et al. [21] to study the crater formation by hypervelocity projectile impact in concrete structures. The authors provided a complete description of the distribution of stresses and strains as functions of time and distance from the center of the cavity, and for a wide range of applied cavity pressures.

Moreover, Buchely and Marañon [22, 23] compared the cavitation fields obtained with the steady-state selfsimilar model developed by Masri and Durban [2] for rate-independent Mises solids with finite element simulations performed for strain-hardening and strain-rate dependent J2 materials. The computations of Buchely and Marañon $[22,23]$ confirmed previous theoretical considerations reported in refs. [15, 19, 20], and showed that the cavitation fields for rate-dependent materials are functions of time, and cannot be modeled with steady-state self-similar approaches. However, Buchely and Marañon [22, 23] limited their attention to relatively low cavity expansion velocities $(m \leq 0.1$, see Eq. (27)) and the effect of material viscosity on shock waves formation at high cavitation velocities was not addressed. Buchely and Marañon [22, 23] also did not pay attention to the effect of stress relaxation that, for viscoplastic materials, plays an important role in the cavitation fields that develop near the cavity.

In an effort to provide further understanding of the effect of material rate-dependence on dynamic cavitation, in this paper we extend the cavity expansion theory developed in refs. $[1,2,3]$ to viscoplastic media. The material behavior is described with an isotropic Perzyna-type overstress formulation $[4,5]$, with associated flow rule, in which the material rate-dependence is controlled by a single viscosity parameter $\eta$. Unlike the self-similar cavity expansion theories, the proposed model has two independent variables: the radial coordinate and the time. The theoretical results, which show the effects of strain-rate dependence and stress relaxation in the stress fields that develop near the cavity, are compared with finite element simulations performed in ABAQUS/Explicit [6]. Excellent agreement between theoretical predictions and numerical results is obtained for the whole range of cavitation velocities investigated $(0.1 \leq m \leq 0.6$, see Eq. (27)). To be highlighted that, in agreement with the results reported by Molinari and Ravichandran [24], both theoretical and finite element results show that material viscosity controls the amplitude and the width of the shock waves that emerge at the highest cavitation velocities investigated. 


\section{Constitutive framework}

We consider an isotropic von Mises material described with an overstress Perzyna-type constitutive model $[4,5]$ that can be represented by the Bingham rheological model shown in Fig. 1, see ref. [25]. The yield function takes the form:

$$
\Phi=\tau_{e}-\tau_{y}
$$

where $\tau_{y}$ is the yield stress, which is assumed to be constant, and $\tau_{e}$ is the effective Kirchhoff stress defined by:

$$
\tau_{e}=\sqrt{\frac{3}{2} s: s}, \quad s=\tau-\tau_{h} \mathbf{1}, \quad \tau_{h}=\frac{1}{3} \boldsymbol{\tau}: \mathbf{1}
$$

where $\boldsymbol{\tau}$ is the Kirchhoff stress tensor, $\boldsymbol{s}$ is its deviatoric part, $\tau_{h}$ is the hydrostatic stress, and $\mathbf{1}$ is the unit second order tensor. We have followed the work of Holzapfel [26] and used the Kirchhoff stress in the formulation of the constitutive equations. Kirchhoff stress appears to be an adequate stress measure to model finite strain metal plasticity, where the observed material hardening mainly results from isochoric dislocation accumulation.

The rate of deformation tensor is taken to be the sum of an elastic, $\boldsymbol{d}^{e}$, and a viscoplastic part, $\boldsymbol{d}^{v p}$ :

$$
\boldsymbol{d}=\boldsymbol{d}^{e}+\boldsymbol{d}^{v p}
$$

The elastic part is related to the rate of the stress by the following hypo-elastic law:

$$
\stackrel{\nabla}{\tau}=C: \boldsymbol{d}^{e}
$$

with $\stackrel{\nabla}{\tau}$ being an objective stress rate and $\boldsymbol{C}$ the isotropic elastic tensor given by:

$$
\boldsymbol{C}=\frac{E}{1+\nu} \boldsymbol{I}^{\prime}+\frac{E}{3(1-2 \nu)} \mathbf{1} \otimes \mathbf{1}
$$

where $E$ is the Young's modulus, $\nu$ is the Poisson's ratio, and $\boldsymbol{I}^{\prime}$ is the unit deviatoric fourth order tensor.

Moreover, assuming an associated flow rule, the viscoplastic part of the rate of deformation tensor is:

$$
\boldsymbol{d}^{v p}=\dot{\lambda} \frac{\partial \tau_{e}}{\partial \boldsymbol{\tau}}
$$

where $\dot{\lambda}$ is a non-negative viscoplastic multiplier given by the following kinetic equation [4]: 


\begin{tabular}{clc}
\hline Symbol & Property and units & Value \\
\hline$\rho_{0}$ & Initial density $\left(\mathrm{kg} / \mathrm{m}^{3}\right)$ & 7800 \\
\hline$E$ & Young's modulus $(\mathrm{GPa})$, Eq. (5) & 210 \\
$\nu$ & Poisson's ratio, Eq. (5) & 0.3 \\
\hline$\tau_{y}$ & Yield stress (MPa), Eq. (1) & 240 \\
$\eta$ & Viscosity parameter $(\mathrm{Pa} \cdot \mathrm{s})$, Eq. (7) & $1.5,150,1500$ \\
\hline
\end{tabular}

Table 1: Material parameters.

$$
\dot{\lambda}=\frac{1}{\eta}\langle\Phi\rangle
$$

where $\eta$ is a viscosity parameter and $\langle x\rangle=\frac{1}{2}(x+|x|)$ is the Macaulay operator.

Moreover, the dissipation consistency:

$$
\boldsymbol{\tau}: \boldsymbol{d}^{v p}=\tau_{e} \dot{\bar{\varepsilon}}^{v p}
$$

leads to the identity:

$$
\dot{\lambda}=\dot{\bar{\varepsilon}}^{v p}
$$

where $\dot{\bar{\varepsilon}}^{v p}$ is the effective viscoplastic strain rate.

The material parameters related to the elastic properties, Eq. (5), the yield stress, Eq. (1), and the viscoplastic kinetic relation, Eq. (7), are given in Table 1. Note that, for the sake of simplicity, we neglect the (potential) strain hardening and thermal softening of the material. Moreover, in Section 5 we carry out a parametric analysis to investigate the effect of viscosity in the cavitation fields. In the parametric analysis different values of $\eta$ are used, see Table 1. We are aware that most metals and alloys present a nonlinear viscoplastic behavior and display values of $\eta$ smaller than $1500 \mathrm{~Pa} \cdot \mathrm{s}$, see for instance ref. [27], where $\eta \approx 280 \mathrm{~Pa} \cdot \mathrm{s}$ for the highest strain rate sensitive branch $\left(\dot{\bar{\varepsilon}}^{v p} \geq 1110 \mathrm{~s}^{-1}\right)$ of a carbon steel. However, considering a wide range of values of $\eta$ helps to bring to light the effect of material viscosity in the cavitation fields and in the formation of shock waves at high cavitation velocities. In addition, assuming that $\eta$ is constant facilitates the interpretation of results. Note that actual materials presenting a nonlinear viscous behavior may be modeled considering a generalization of the viscoplastic framework used in this work. For this purpose, the function $\langle\Phi\rangle$ should be modified accordingly (e.g. refs. [28, 29, 30]). Nevertheless, including nonlinear viscosity in the theoretical and finite element models is left for a future work.

\section{Theoretical model}

In this section we extend the dynamic spherical cavity expansion model developed in refs. [1, 2, 3] to viscoplastic materials. We also incorporate into the model the artificial viscosity approach used in refs. [31, 18] to capture the 
formation of plastic shock waves at high cavitation velocities in rate-independent materials. The goal is to study whether this artificial viscosity is required to model shocks in viscoplastic media or if, on the contrary, the material viscosity is sufficient to regularize the problem and describe the emergence of shocks.

Consider a pressurized spherical cavity of instantaneous radius $a$ expanding at constant velocity in an infinite medium. We denote $(r, \theta, \phi)$ the radial, circumferential and azimuthal Eulerian coordinates of a material point with reference to a spherical system with the origin located at the center of the cavity $O$, see Fig. 1 . We introduce the dimensionless variable $\xi=r / a$ such that the derivative with respect to the radial coordinate is:

$$
\frac{\partial()}{\partial r}=\frac{\partial()}{\partial \xi} \frac{\partial \xi}{\partial r}=()^{\prime} \frac{1}{a}
$$

where the prime superscript denotes differentiation with respect to $\xi$. Moreover, the time derivative is [14]:

$$
\dot{()}=\frac{\partial()}{\partial \xi} \dot{\xi}=()^{\prime} \frac{\dot{a}}{a}(v-\xi)
$$

where $v=\dot{r} / \dot{a}$ is the dimensionless radial velocity. Since $\dot{a}$ is constant (constant-velocity expansion), the dimensionless acceleration is $\dot{v}=\ddot{r} / \dot{a}$.

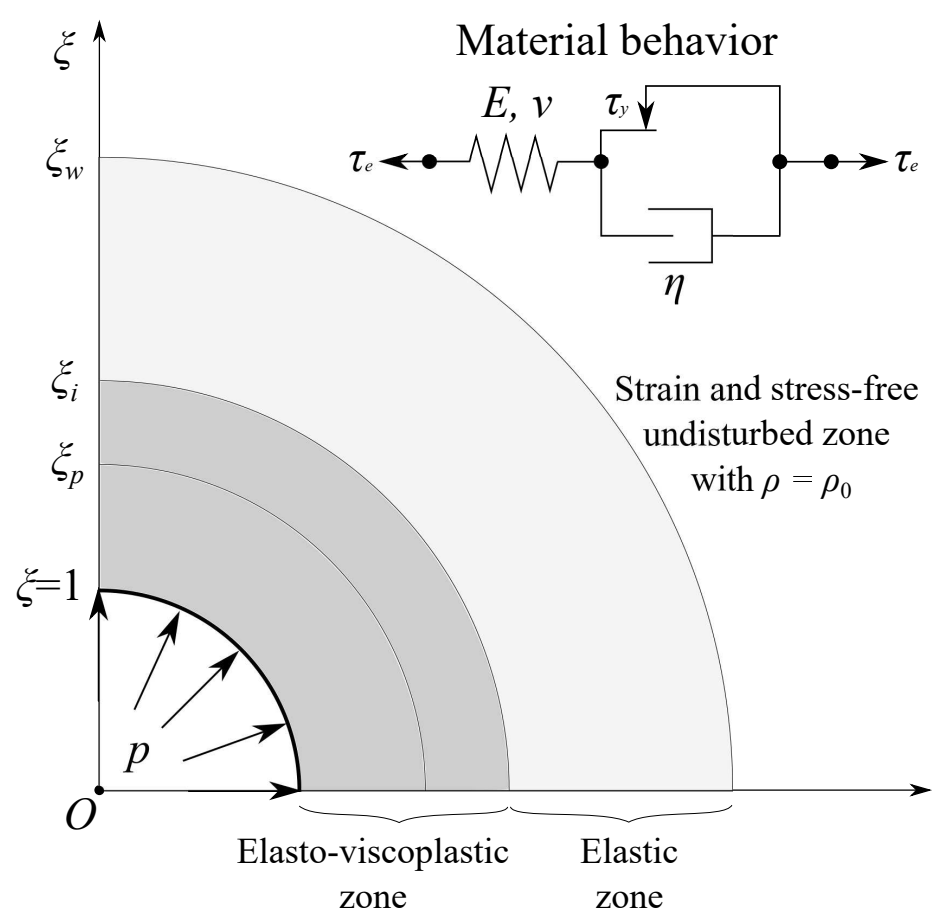

Figure 1: Scheme of the cavitation field induced by a pressurized spherical cavity expanding dynamically in an elasto-viscoplastic medium. The internal pressure $p$ is applied at the cavity wall where the dimensionless radial coordinate is $\xi=r / a=1$. The remote field, at $\xi>\xi_{w}$, is undisturbed. Behind the elastic wave front, located at $\xi=\xi_{w}$, an elastic range develops. The transition between the elastic and elasto-viscoplastic regions is denoted by $\xi_{i}$, and the location of the viscoplastic shock wave by $\xi_{p}$ (a viscoplastic shock wave emerges at high cavitation velocities). The material behaviour can be represented by the Bingham rheological model shown in the upper right part of the illustration. This figure is adapted from Durban and Masri [1]. 
For spherical deformations, and isotropic material behavior, the non-zero components of the Kirchhoff stress tensor are $\tau_{r r}$ and $\tau_{\theta \theta}=\tau_{\phi \phi}$, which leads to the following expression for the effective Kirchhoff stress:

$$
\tau_{e}=\tau_{\theta \theta}-\tau_{r r}
$$

Similarly, the non-zero components of the rate of deformation tensor are:

$$
\begin{gathered}
d_{r r}=\frac{\partial \dot{r}}{\partial r} \\
d_{\theta \theta}=d_{\phi \phi}=\frac{\dot{r}}{r}
\end{gathered}
$$

The elastic strain rates, Eq. (4), can be rewritten as:

$$
\begin{gathered}
d_{r r}^{e}=\frac{1}{E} \dot{\tau}_{r r}-\frac{2 \nu}{E} \dot{\tau}_{\theta \theta} \\
d_{\theta \theta}^{e}=d_{\phi \phi}^{e}=\frac{1-\nu}{E} \dot{\tau}_{\theta \theta}-\frac{\nu}{E} \dot{\tau}_{r r}
\end{gathered}
$$

In previous expressions, since the material spin is zero, the objective derivative $\stackrel{\nabla}{()}$ (see Eq. (4)) has been replaced by a time derivative $(\dot{)}$.

Moreover, the viscoplastic strain rates, Eq. (6), can be rewritten as:

$$
\begin{gathered}
d_{r r}^{v p}=-\dot{\bar{\varepsilon}}^{v p} \\
d_{\theta \theta}^{v p}=d_{\phi \phi}^{v p}=\frac{1}{2} \dot{\bar{\varepsilon}}^{v p}
\end{gathered}
$$

Using equations (10)-(18), the additive decomposition of the rate of deformation tensor, Eq. (3), leads to the following two expressions for the radial and circumferential directions:

$$
\begin{gathered}
v^{\prime}=(v-\xi)\left[(1-2 \nu) T_{r r}-2 \nu T_{e}-\bar{\varepsilon}^{v p}\right]^{\prime} \\
\frac{v}{\xi}=(v-\xi)\left[(1-2 \nu) T_{r r}+(1-\nu) T_{e}+\frac{1}{2}\left(\bar{\varepsilon}^{v p}\right)\right]^{\prime}
\end{gathered}
$$


where $T_{r r}=\tau_{r r} / E$ and $T_{e}=\tau_{e} / E$ are the dimensionless radial and effective Kirchhoff stresses, respectively, and $\bar{\varepsilon}^{v p}=\int_{0}^{t} \dot{\bar{\varepsilon}}^{v p} d \gamma$ is the effective viscoplastic strain, where $t$ refers to time.

Moreover, during plastic flow, we have that $\Phi \geq 0$, which allows to rewrite the kinetic equation (7) as:

$$
(v-\xi)\left(\bar{\varepsilon}^{v p}\right)^{\prime}=\frac{a}{t^{*} \dot{a}}\left(T_{e}-T_{y}\right)
$$

where equations (1) and (11) have been used. For a constant-velocity expansion, i.e. $\dot{a}=$ constant, the current cavity radius can be calculated as $a=a_{0}+\dot{a} t$, where $a_{0}$ is the cavity radius at $t=0$ (initial cavity radius). Therefore, the dynamic cavity expansion model developed in this paper is time-dependent, thus requiring to specify the time $t$ at which the corresponding cavitation fields are evaluated $[15,19]$. Moreover, in previous expression $t^{*}=\eta / E$ is the so-called relaxation time [5] and $T_{y}=\tau_{y} / E$ is the dimensionless yield stress. Equation (21) shows that the rate-dependence of this problem is inversely proportional to the cavity radius $a$, leading to stress relaxation as the cavity enlarges (see Section 5). For the limiting cases in which $a \rightarrow \infty$ (i.e. $t \rightarrow \infty$ ) or $t^{*} \rightarrow 0$ (i.e. $\eta \rightarrow 0$, rate-independent material), the cavitation fields become steady-state and self-similar.

Moreover, the ratio between the current and initial material densities can be obtained from the mass conservation equation which, in Eulerian description, yields:

$$
\dot{\rho}+\rho \operatorname{tr}(\boldsymbol{l})=0
$$

where $\boldsymbol{l}$ is the spatial velocity gradient. In absence of material spin $\boldsymbol{l}=\boldsymbol{d}$, and, using equations (11), (13) and (14), previous expression can be rewritten as:

$$
(v-\xi) \frac{\rho^{\prime}}{\rho}+v^{\prime}+2 \frac{v}{\xi}=0
$$

Inserting (19) and (20) into equation (23), the following expression is obtained:

$$
\frac{\rho^{\prime}}{\rho}=-3(1-2 \nu) T_{h}^{\prime}
$$

where $T_{h}=\tau_{h} / E$ is the dimensionless hydrostatic stress. Integrating equation (24) from a given coordinate $\xi$ to the elastic wave front $\xi_{w}$, where $\rho=\rho_{0}$ and $T_{h}=0$ (see Fig. 1), the following relation for the density ratio is obtained:

$$
J^{-1}=\exp \left[-3(1-2 \nu) T_{h}\right]
$$

where $J=\rho_{0} / \rho$ is the material Jacobian (i.e. the determinant of the deformation gradient tensor). 
Moreover, the balance of linear momentum along the radial direction, in Eulerian description, takes the form:

$$
\frac{\partial\left(J^{-1} \bar{\tau}_{r r}\right)}{\partial r}+\frac{2 J^{-1}}{r}\left(\bar{\tau}_{r r}-\bar{\tau}_{\theta \theta}\right)=\rho \ddot{r}
$$

where $\bar{\tau}_{r r}$ and $\bar{\tau}_{\theta \theta}$ are the total radial and circumferential Kirchhoff stresses given by the sum of the elasto-viscoplastic stresses and the viscous stresses, i.e. $\bar{\tau}_{r r}=\tau_{r r}+\tau_{r r}^{v}$ and $\bar{\tau}_{\theta \theta}=\tau_{\theta \theta}+\tau_{\theta \theta}^{v}$. On the one hand, $\tau_{r r}$ and $\tau_{\theta \theta}$ are related to the elastic and viscoplastic deformation rates by the constitutive equations presented in Section 2 (i.e. equations (15)-(18) in this Section). On the other hand, $\tau_{r r}^{v}$ and $\tau_{\theta \theta}^{v}$ are related to the total deformation rates by means of an artificial viscosity [32] to be presented in the sequel. Using equations (10) and (11), and the relation $J=\rho_{0} / \rho$, the linear momentum equation (26) can be rewritten as:

$$
\bar{T}_{r r}^{\prime}+J \bar{T}_{r r}\left(J^{-1}\right)^{\prime}-\frac{2}{\xi} \bar{T}_{e}=m^{2}(v-\xi) v^{\prime}
$$

where $\bar{T}_{r r}=\frac{\bar{\tau}_{r r}}{E}$ and $\bar{T}_{e}=\frac{\bar{\tau}_{e}}{E}$ are dimensionless total Kirchhoff stresses, and $m=\dot{a} / \sqrt{E / \rho_{0}}$ is the dimensionless cavitation velocity defined as the ratio between the cavity expansion velocity $\dot{a}$ and the elastic wave velocity in a long rod $\sqrt{E / \rho_{0}}$. Note that the artificial viscosity approach is only used in the definition of the stresses that enters into the balance of linear momentum (see refs. [31, 18]).

Following the development presented in refs. $[33,32,31,18]$, the dimensionless viscous Kirchhoff stresses, $T_{r r}^{v}=\tau_{r r}^{v} / E$ and $T_{\theta \theta}^{v}=\tau_{\theta \theta}^{v} / E$, are calculated as:

$$
T_{r r}^{v}= \begin{cases}-\eta_{r r} m\left|v^{\prime}\right| & \text { if } v^{\prime}<0 \\ 0 & \text { if } v^{\prime} \geq 0\end{cases}
$$

and

$$
T_{\theta \theta}^{v}= \begin{cases}-\eta_{\theta \theta} m|v| & \text { if } v<0 \\ 0 & \text { if } v \geq 0\end{cases}
$$

being

$$
\eta_{r r}=c_{0}^{2}(\Delta \xi)^{2} \frac{\rho}{\rho_{0}} m\left|v^{\prime}\right|+c_{L} \Delta \xi \sqrt{\frac{\rho}{\rho_{0}}}
$$

and

$$
\eta_{\theta \theta}=c_{0}^{2}\left(\frac{\Delta \xi}{\xi}\right)^{2} \frac{\rho}{\rho_{0}} m|v|+c_{L} \frac{\Delta \xi}{\xi} \sqrt{\frac{\rho}{\rho_{0}}}
$$

the radial and circumferential artificial viscosity parameters, respectively. In equations (30) and (31), $c_{0}$ and $c_{L}$ are 
constant parameters (their values are given in Section 5.2), and $\Delta \xi$ is a given increment along the dimensionless radial coordinate $\xi$. Recall that in refs. $[31,18]$ the artificial viscosity was essential to model plastic shocks in rate-independent materials. In this paper, as mentioned before, the idea is to investigate if the artificial viscosity is necessary to model shock waves in viscoplastic media. Moreover, to identify the emergence of a shock we use the approach developed in ref. [3] for rate-independent materials. Namely, we assume that a shock wave is formed if the following condition is met:

$$
m^{2}(v-\xi)^{2}+T_{r r}-\frac{1}{3(1-2 \nu)}=0
$$

All the details of the derivation of expression (32) are given in ref. [3].

In the dynamic cavity expansion model developed in this paper, equations (19), (20), (21), (25), (27) and (28), provide a system of five differential equations and one algebraic relation with derivatives of six unknowns $\left(v, \bar{\varepsilon}^{v p}, J, T_{r r}, T_{r r}^{v}, T_{e}\right)$, with $T_{\theta \theta}^{v}$ being calculated from the algebraic relation (29). Integration is performed over the dimensionless radial coordinate $\xi$, from the elastic wave front $\xi_{w}$ up to the cavity wall $\xi=1$. The following boundary conditions have to be satisfied:

$$
v=1, \quad T_{r r}=-J P, \quad \text { at } \xi=1
$$

and

$$
v=0, \quad T_{r r}=T_{e}=0, \quad J=1, \quad \text { at } \xi=\xi_{w}
$$

where $P=p / E$ is the dimensionless applied pressure (assumed constant, see Fig. 1). The compatibility of the field variables at the interface between elastic and elasto-viscoplastic zones (see Fig. 1), $\xi=\xi_{i}$, has been enforced using the closed-form elastic solution derived in ref. [1]. The problem is solved using the numerical integration scheme proposed in refs. $[1,2,3]$.

\section{Finite element model}

This section describes the main features of the axisymmetric finite element model developed in ABAQUS/Explicit [6] to simulate dynamic spherical cavitation. This same model has been used in refs. [34, 31]. The specimen is a sphere with radius $R_{s}=300 \mathrm{~mm}$ which has a small cavity of radius $R_{c}=0.5 \mathrm{~mm}$ in its center. To reduce the computational cost of the simulations, only the $\theta \geq 0$ half of the specimen has been analyzed (see Fig. 2). The sample is initially at rest and undeformed, while a constant internal pressure $p$ is applied at the cavity wall (consistently with the theoretical model, see Fig. 1). Note that the dimensions of the sphere and the cavity are taken such that, for all the numerical simulations, the stress waves generated by the application of the cavity pressure reach the 


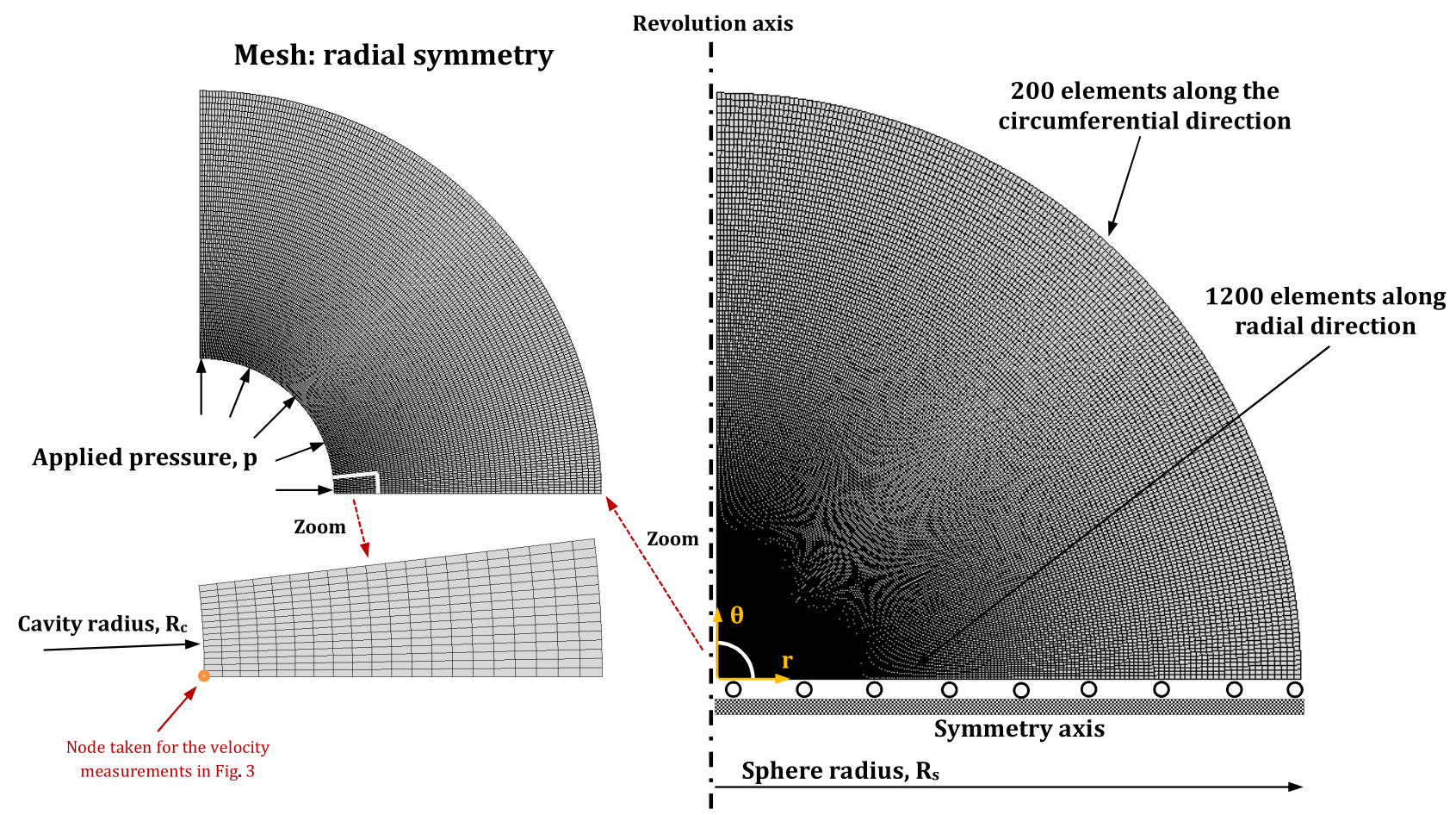

Figure 2: Axisymmetric finite element model developed in ABAQUS/Explicit [6] to simulate dynamic spherical cavitation. This figure is adapted from refs. [34, 31].

external free boundary of the sphere after the cavity has started to expand at constant speed (i.e. cavitation has occurred [34]).

The model has been meshed with 240,000 four-node axisymmetric reduced integration elements, CAX4R in ABAQUS notation. The mesh shows radial symmetry, in order to retain the symmetry of the problem, with 200 elements along the circumferential direction and 1200 along the radial direction. The elements size is constant along the circumferential direction whereas it decreases along the radial direction as the cavity is approached with a bias ratio of 100 . The dimensions of the elements located near the cavity are $12 \mu \mathrm{m} \times 4 \mu \mathrm{m}$. Large gradients of stress and strain arise near the cavity, and within the shock wave that emerges at high cavitation velocities, which may lead to severe mesh distortion. In order to circumvent this problem, the Arbitrary Lagrangian Eulerian (ALE) adaptive meshing available in ABAQUS/Explicit [6] has been used, see refs. [34, 31]. Moreover, note that ABAQUS/Explicit [6] introduces artificial damping in the calculations in order to attenuate the numerical solution and ensure stability, see ref. [35]. The code generates bulk viscosity pressures, which are linear and quadratic, respectively, in the volumetric strain rate. This artificial viscosity is controlled by two parameters $\varpi$ (linear viscosity) and $\chi$ (quadratic viscosity). In the calculations shown in Section 5 , the default values $\varpi=0.06$ and $\chi=1.2$ have been used.

For the implementation of the constitutive model presented in Section 2 in ABAQUS/Explicit [6] a user material subroutine VUMAT was developed. The integration of the constitutive equations has been performed using the 
radial return mapping algorithm, see refs. [36, 37].

\section{Data analysis and discussion}

This section is split into two parts. In Section 5.1, the finite element simulations are used to identify the time required to reach the constant-velocity expansion of the cavity, for a wide range of dimensionless cavitation velocities $0.1 \leq m \leq 0.6$, and the three values of the viscosity parameter given in Table 1 . Similar range of values of $m$ was investigated in refs. [16, 31, 18]. In Section 5.2, the elasto-viscoplastic cavitation fields predicted by the theoretical model are compared with the finite element results for different loading times.

\subsection{Key finite element results}

Figure 3 shows the radial velocity of a node located at the cavity wall $\dot{a}$ versus the loading time $t$ for finite element simulations performed using three values of the viscosity parameter $\eta$, namely: 1.5, 150 and $1500 \mathrm{~Pa} \cdot \mathrm{s}$ (see Table 1). The node taken for the velocity measurements is indicated in Fig. 2. Results are reported for four different applied pressures, $p=3696,10479,36645$ and $78351 \mathrm{MPa}$, such that the corresponding cavitation velocities are $\dot{a} \approx 520,1040,2080$ and $3120 \mathrm{~m} / \mathrm{s}$, respectively (Figs. 3(a), 3(b), 3(c) and 3(d)). These cavitation velocities correspond to $m=0.1,0.2,0.4$ and 0.6 (recall from Section 3 that $m=\dot{a} / \sqrt{E / \rho_{0}}$ ). Note that, for a given applied pressure, the cavitation velocity is virtually independent of the material viscosity. This behaviour is, most likely, due to the fact that for low values of $m$ the influence of viscosity in the cavitation fields is small, and for large values of $m$ the stress state is nearly hydrostatic, see Section 5.2. Note also that the relationship between the applied pressure and the cavitation velocity is nonlinear, in agreement with the results reported in refs. [16, 31, 18] for rate-independent materials. Moreover, the $\dot{a} \times t$ curves display a concave-downward shape, for the four values of $m$, and the three viscosity parameters considered. The rate of increase of the expansion velocity slows down as $t$ increases such that $\dot{a}$ eventually becomes constant, indicating the onset of cavitation. The time to reach the constant-velocity expansion, hereinafter denoted by $t^{c a v}$, and referred to as cavitation time, is taken as the loading time for which the condition $\ddot{a} \leq 1 \times 10^{6} \mathrm{~m} / \mathrm{s}^{2}$ is met. The condition $\ddot{a} \leq 1 \times 10^{6} \mathrm{~m} / \mathrm{s}^{2}$ is chosen because for this value of $\ddot{a}$ the results for the three viscosity parameters investigated virtually overlap each other.

Fig. 4 shows the cavitation time $t^{\text {cav }}$ versus the dimensionless cavitation velocity $m$, for the three values of $\eta$ investigated. The cavitation time $t^{c a v}$ decreases nonlinearly with $m$ (see also refs. [34, 31]), such that the $t^{c a v} \times m$ curves display a concave-upward shape, with faster decrease of $t^{c a v}$ for short values of $m$. Moreover, the cavitation time is greater as the material viscosity increases, i.e. the $t^{c a v} \times m$ curve is shifted upwards as the value of $\eta$ increases for the whole range of cavitation velocities investigated. The influence of viscosity in the cavitation time is greater for small values of $m$. The cavitation times obtained for $\eta=1500 \mathrm{~Pa} \cdot \mathrm{s}$ are from 2 to 7 times greater than those obtained for $\eta=1.5 \mathrm{~Pa} \cdot \mathrm{s}$ and $\eta=150 \mathrm{~Pa} \cdot \mathrm{s}$. It becomes apparent that material viscosity delays cavitation, extending the transient behavior that precedes the constant-velocity expansion of the cavity. Moreover, note that 


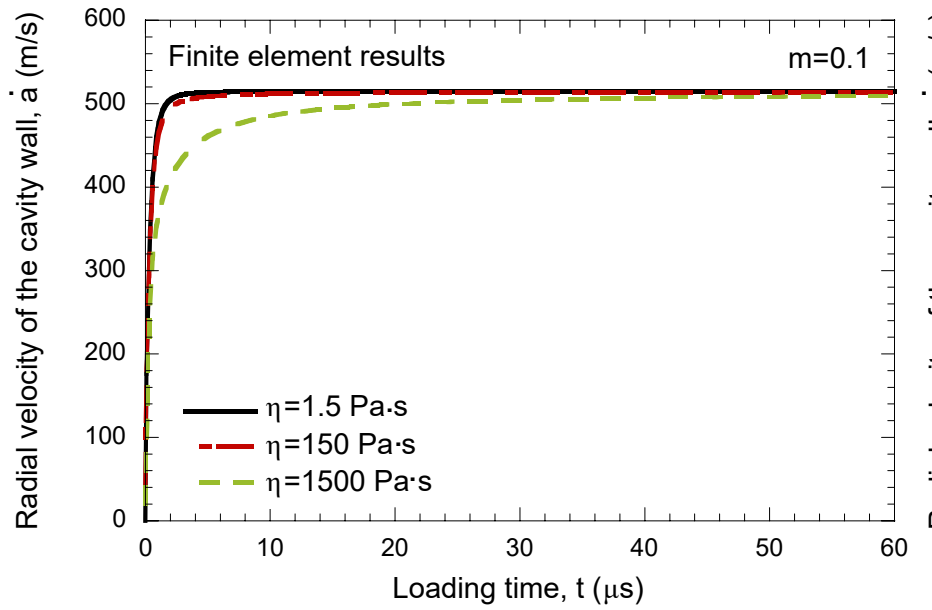

(a)

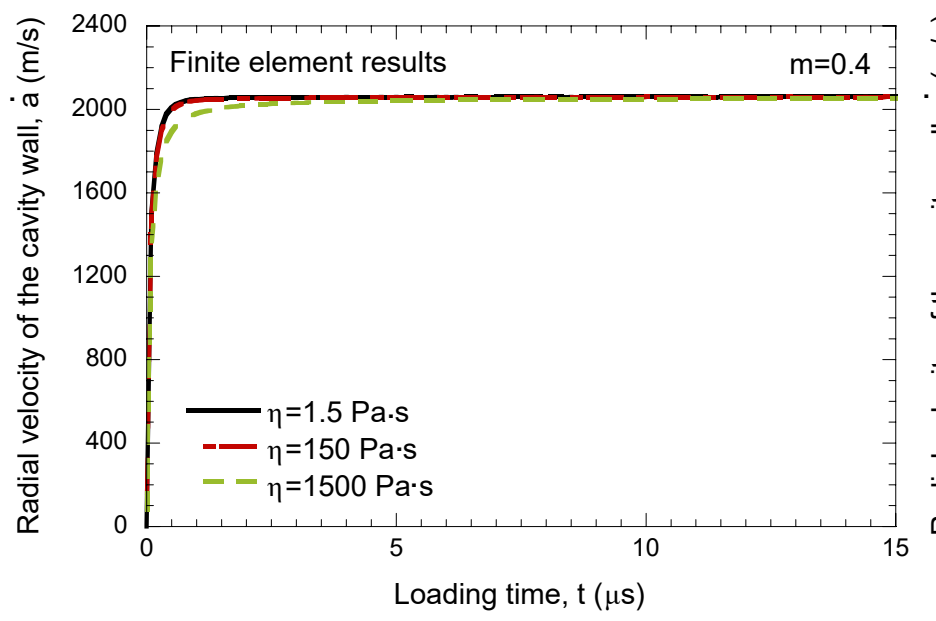

(c)

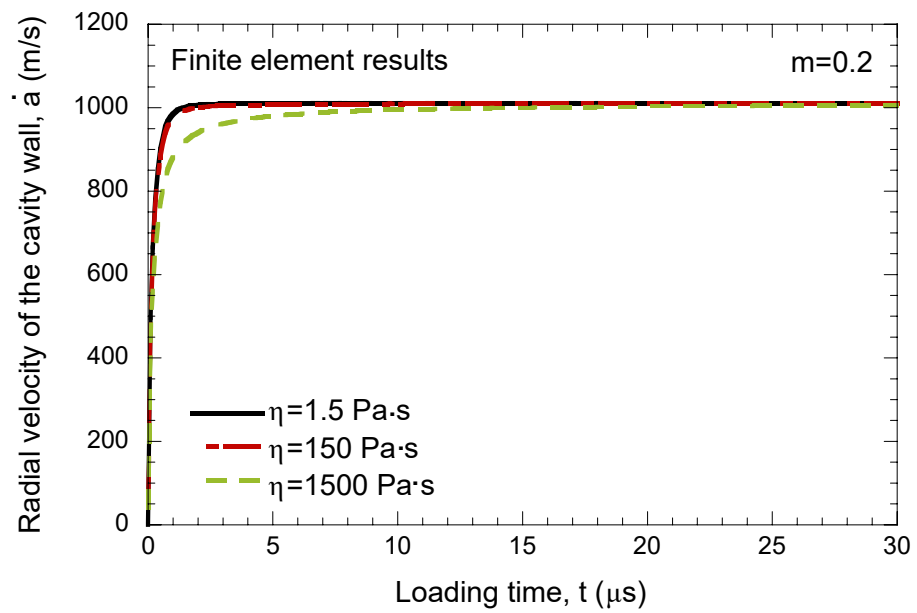

(b)

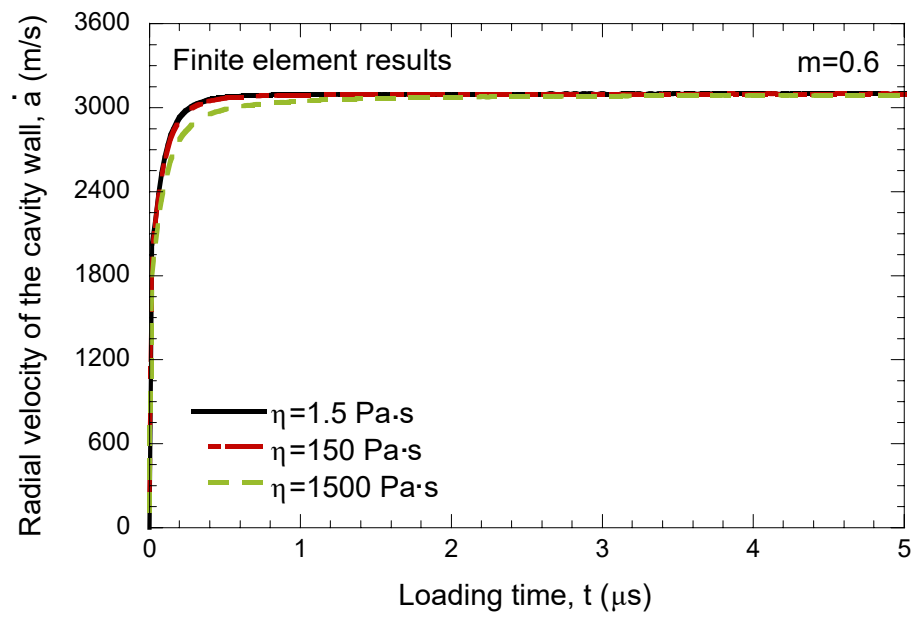

(d)

Figure 3: Finite element results. Radial velocity of a node located at the cavity wall $\dot{a}$ versus the loading time $t$ for three values of the viscosity parameter $\eta=1.5,150$ and $1500 \mathrm{~Pa} \cdot \mathrm{s}$. Results are shown for four different dimensionless cavitation velocities: (a) $m=0.1$, (b) $m=0.2$, (c) $m=0.4$, (d) $m=0.6$. 


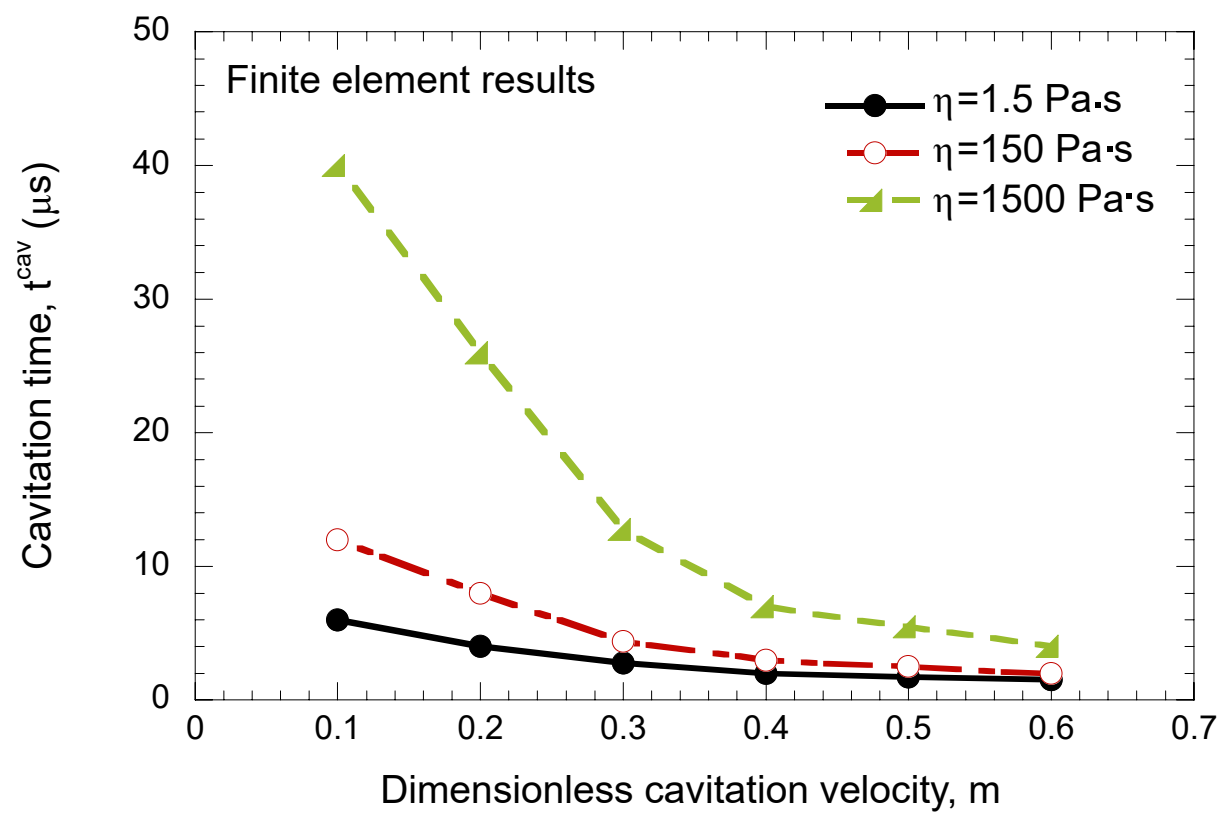

Figure 4: Finite element results. Cavitation time $t^{\text {cav }}$ versus dimensionless cavitation velocity $m$ for three values of the viscosity parameter $\eta=1.5,150$ and $1500 \mathrm{~Pa} \cdot \mathrm{s}$.

in ref. [34] it was stated that, if the penetration process is much faster than the time required for constant-velocity cavity expansion to appear, then constant-velocity cavitation fields are not sufficient to describe the underlying physical phenomena. Therefore, the results presented in this paper, which reveal that viscosity delays cavitation, suggest that the increase of material rate-dependence may hinder the application of constant-velocity cavitation fields to model high-velocity penetration problems. Nevertheless, this is still an open issue that requires further research.

\subsection{Verification of the theoretical model}

The verification is performed comparing the stress fields predicted by the theoretical model with the finite element results. The comparison is carried out for loading times greater than $t^{\text {cav }}$, for which the constant-velocity expansion solution developed in Section 3 is valid. Moreover, despite that the Kirchhoff stress tensor $\tau$ was used to formulate the constitutive framework and the theoretical model (see Sections 2 and 3), with the aim of facilitating the interpretation of the results, since the most commonly used measure of stress is the Cauchy stress tensor $\boldsymbol{\sigma}$, the stress fields shown in the sequel are given in terms of the components of the dimensionless Cauchy stress tensor $\boldsymbol{\Sigma}=\sigma / E$. The tensor $\boldsymbol{\Sigma}$ is readily calculated as $\boldsymbol{\Sigma}=J^{-1} \boldsymbol{T}$, where $\boldsymbol{T}=\tau / E$ is the dimensionless Kirchhoff stress tensor (see Section 3). Moreover, note that all the theoretical stress fields correspond to the elasto-viscoplastic stresses (see Section 3). The viscous stresses lack of physical meaning, and their role in the theoretical model is limited to facilitate the integration of the governing equations when shock waves appear at high cavitation velocities and the viscosity of the material is low $[31,18]$. If the material viscosity is high, the artificial viscosity is no longer needed to model the formation of shocks, as will be discussed further below. 
Figure 5 compares theoretical (TM) and finite element results (FEM) for $m=0.1$. This low cavitation velocity does not lead to the emergence of shock waves, the artificial viscosity was not necessary to obtain the theoretical predictions, and the parameters $c_{0}$ and $c_{L}$ were set to zero (see Section 3 ). Recall from Section 3 that we consider a shock wave to form when the condition (32) is met.

Figures 5(a)-(b) show the evolution of the dimensionless radial Cauchy stress $\Sigma_{r r}$ versus the dimensionless radial coordinate $\xi$ for $\eta=1.5 \mathrm{~Pa} \cdot \mathrm{s}$ and $\eta=1500 \mathrm{~Pa} \cdot \mathrm{s}$, respectively. Note that the material viscosity hardly influences the radial stress fields. The $\Sigma_{r r} \times \xi$ curves, as they are displayed in this paper with increasing negative values of the radial stress along the $y$-axis, show a concave-upward shape, with the maximum value of the radial stress $\Sigma_{r r}=-0.0177$ (compression) located at the cavity wall $\xi=1$, and the elastic wave front located at $\xi_{w}=11.60$. The agreement between theoretical predictions and finite element results is very good. In addition, the results reported for different loading times, for both viscosity parameter values, virtually overlap each other, which indicates that the radial stress fields are nearly steady-state and self-similar. Namely, for $\eta=1.5 \mathrm{~Pa} \cdot \mathrm{s}$ results are shown for $t=10 \mu \mathrm{s}$ and $20 \mu \mathrm{s}$, and for $\eta=1500 \mathrm{~Pa} \cdot \mathrm{s}$ the loading times considered are $t=40 \mu \mathrm{s}, 50 \mu \mathrm{s}$ and $t \rightarrow \infty$ (only theoretical predictions). Recall that the limiting case of $t \rightarrow \infty$ corresponds to the rate-independent material response. Moreover, note that, as anticipated, the loading times are equal or greater than the cavitation times $t^{c a v}$ reported in Fig. 4. Namely, the smaller loading times considered correspond, approximately, to the cavitation time, see Fig. 4.

Figures 5(c)-(d) show the variation of the dimensionless effective Cauchy stress $\Sigma_{e}$ with the dimensionless radial coordinate $\xi$ for the same two values of the viscosity parameter. Note that, near the cavity, the effective stress is one order of magnitude smaller than the absolute value of the radial stress. On the other hand, as in the case of the radial stress fields, the agreement between theoretical predictions and finite element results is very good. For $\eta=1.5 \mathrm{~Pa} \cdot \mathrm{s}$, Fig. 5(c), the effective stress slightly decreases as we move away from the cavity wall until the elastic/elasto-viscoplastic interface is reached at $\xi_{i}=6.04$. For $\xi_{i}<\xi<\xi_{w}$, which corresponds to the elastic zone, the effective stress $\Sigma_{e}$ shows a steeper decline, featuring a concave-upward shape. Note that the results obtained for the two loading times considered, $t=10 \mu \mathrm{s}$ and $t=20 \mu \mathrm{s}$, are very similar, showing that the effective stress fields, for this value of the viscosity parameter, are nearly steady-state and self-similar. In contrast, for $\eta=1500 \mathrm{~Pa} \cdot \mathrm{s}$, Fig. 5(d), there are meaningful differences between the results obtained near the cavity for different loading times. The effective stress decreases as the loading time increases, i.e. for this value of the viscosity parameter the relaxation of the material as the cavity increases influences the effective stress fields. The decrease of the effective Cauchy stress with the radial coordinate within the elasto-viscoplastic zone for $t \rightarrow \infty$ (rate-independent limit) is due to the influence of material compressibility in $\Sigma_{e}$. Recall that the yield criterion is formulated in terms of the Kirchhoff stress, see Eq. (1).

Figure 6 compares the theoretical and finite element stress fields for a greater cavity expansion velocity $m=0.2$. 


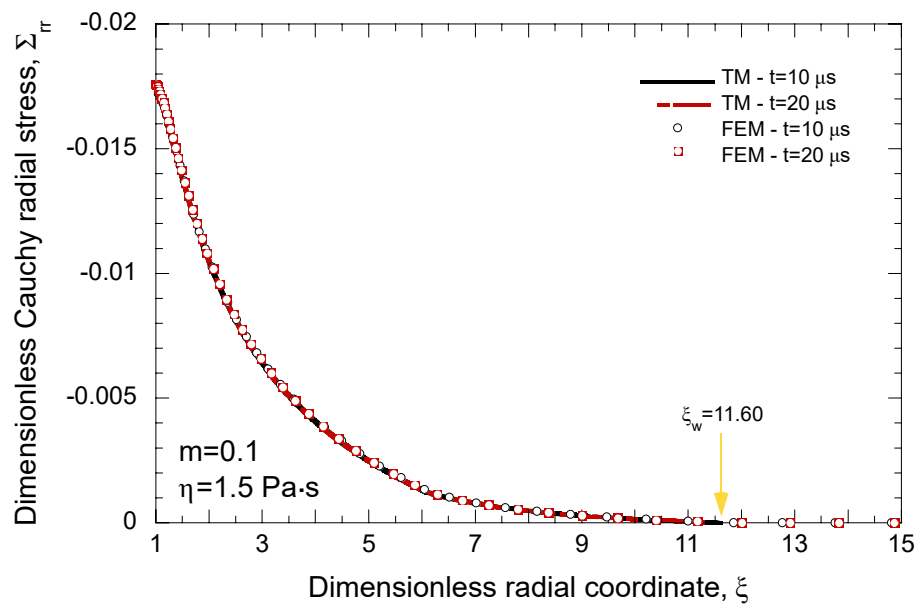

(a)

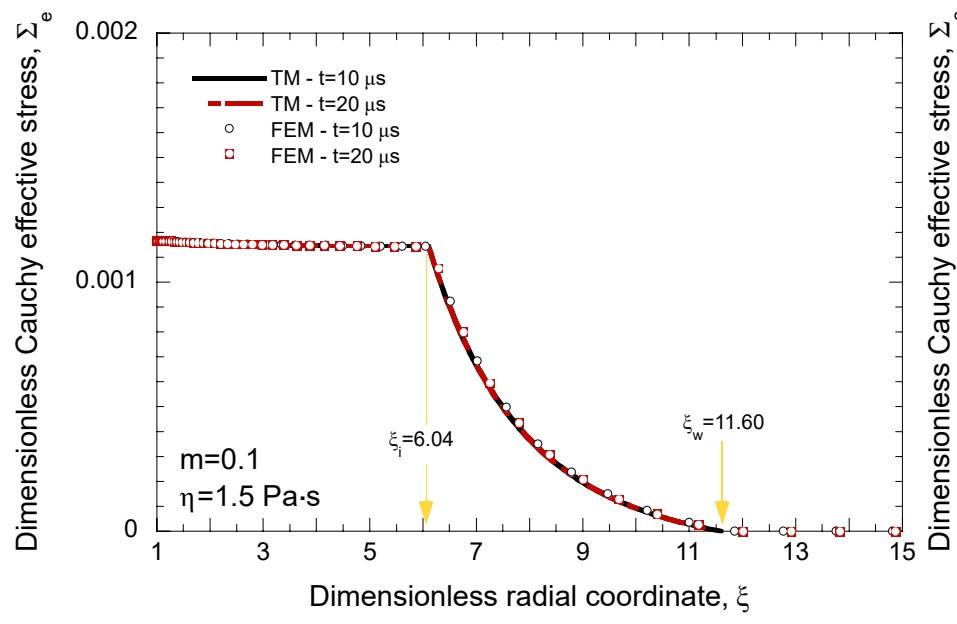

(c)

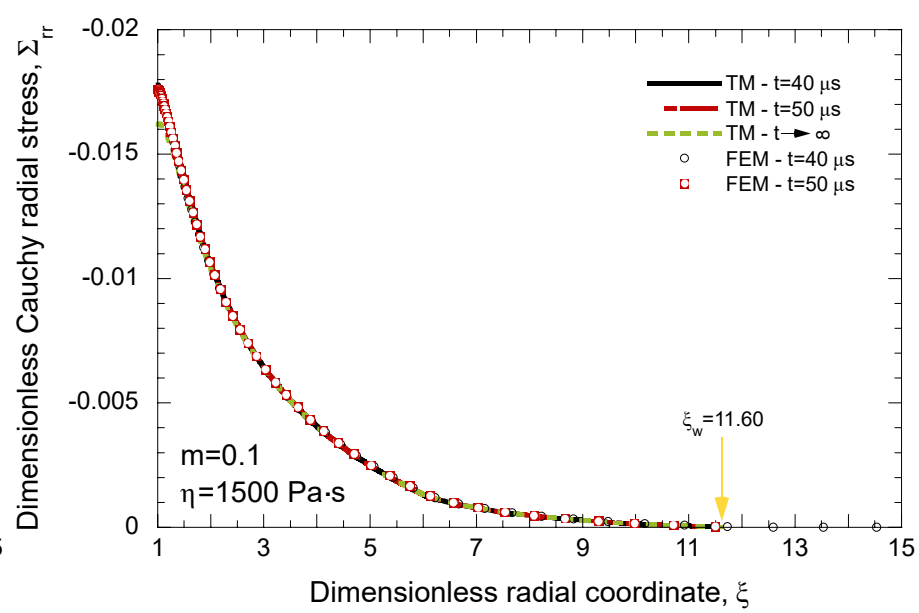

(b)

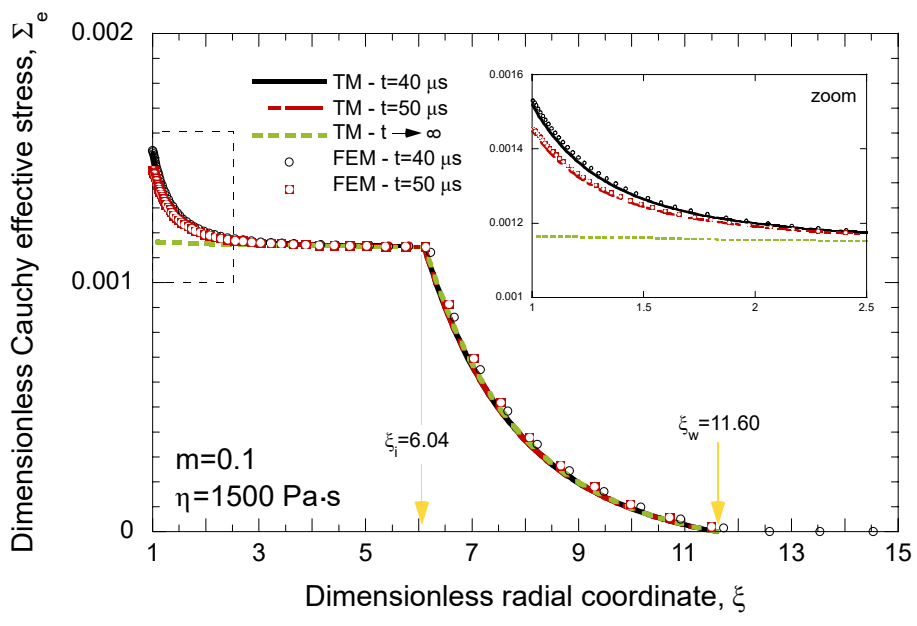

(d)

Figure 5: Comparison between theoretical results (TM) and finite element results (FEM) for $m=0.1$. Dimensionless radial Cauchy stress $\Sigma_{r r}$ versus dimensionless radial coordinate $\xi$ for (a) $\eta=1.5 \mathrm{~Pa} \cdot \mathrm{s}$ and (b) $\eta=1500 \mathrm{~Pa} \cdot \mathrm{s}$. Dimensionless effective Cauchy stress $\Sigma_{e}$ versus dimensionless radial coordinate $\xi$ for (c) $\eta=1.5 \mathrm{~Pa} \cdot \mathrm{s}$ and (d) $\eta=1500 \mathrm{~Pa} \cdot \mathrm{s}$. The theoretical results were obtained without artificial viscosity, $c_{0}=0$ and $c_{L}=0$. 
This cavitation velocity neither leads to the emergence of shocks (the condition (32) is not met), so that we set $c_{0}=0$ and $c_{L}=0$ in the theoretical model. As in Fig. 5, results are shown for $\eta=1.5 \mathrm{~Pa} \cdot \mathrm{s}$ and $\eta=1500 \mathrm{~Pa} \cdot \mathrm{s}$. The agreement between theoretical and finite element calculations is very good.

Figures 6(a)-(b) show the evolution of the radial Cauchy stress $\Sigma_{r r}$ versus the dimensionless radial coordinate $\xi$. The influence of the material viscosity on the $\Sigma_{r r} \times \xi$ curves is very small. Moreover, the radial stress fields obtained for different loading times practically overlap each other. The difference with the $\Sigma_{r r} \times \xi$ curves shown in Figs. 5(a)-(b) is that the absolute value of the radial stress near the cavity is $\approx 3$ times greater.

The evolution of the effective Cauchy stress $\Sigma_{e}$ with the dimensionless radial coordinate $\xi$ is displayed in Figs. 6(c)-(d). Compared to the results reported in Figs. 5(c)-(d), the $\Sigma_{e} \times \xi$ curves for $m=0.2$ show increased gradients of effective stress near the cavity. The increase of the cavitation velocity leads to greater strain rates and greater material compressibility near the cavity wall, which boosts the effective Cauchy stress. The greater the cavitation velocity and the material viscosity, the more important the effective Cauchy stress gradients near the cavity are. Moreover, note that in the case of $\eta=1500 \mathrm{~Pa} \cdot \mathrm{s}$, Fig. 6(d), the stress relaxation leads to significant differences in the effective stress near the cavity for the loading times considered, which illustrates that the material viscosity leads to time-dependent cavitation fields [38]. As the loading time increases, the gradients of stress in the elastoviscoplastic zone get gradually reduced. As mentioned before, the decrease of the effective Cauchy stress near the cavity for $t \rightarrow \infty$ (rate-independent limit) is due to the influence of material compressibility in $\Sigma_{e}$.

Figures 7 and 8 show the theoretical and finite element stress fields for $m=0.4$ and three values of the viscosity parameter $\eta=1.5,150$ and $1500 \mathrm{~Pa} \cdot \mathrm{s}$. This cavitation velocity leads to the formation of a shock wave. The minimum value of $m$ for which the shock condition (32) is met is 0.34 for the three viscosity parameters (recall that the shock condition was developed for rate-independent materials). Moreover, in the case of $\eta=1.5 \mathrm{~Pa} \cdot \mathrm{s}$, the theoretical results were obtained using the artificial viscosity with $c_{0}=0.55$ and $c_{L}=0.25$. In contrast, for $\eta=150 \mathrm{~Pa} \cdot \mathrm{s}$ and $\eta=1500 \mathrm{~Pa} \cdot \mathrm{s}$, the theoretical solution could be obtained without artificial viscosity because the large material viscosity facilitated the integration of the governing equations for the loading times investigated. However, for larger loading times, $t>22 \mu \mathrm{s}$ and $t>226 \mu \mathrm{s}$, due to stress relaxation, the artificial viscosity becomes also necessary to capture the shock wave for $\eta=150 \mathrm{~Pa} \cdot \mathrm{s}$ and $\eta=1500 \mathrm{~Pa} \cdot \mathrm{s}$, respectively. Moreover, note that for this cavitation velocity, the theoretical model also reproduces very well the finite element results.

The evolution of the radial Cauchy stress $\Sigma_{r r}$ versus the dimensionless radial coordinate $\xi$ for $\eta=1.5,150$ and $1500 \mathrm{~Pa} \cdot \mathrm{s}$ is shown in Figs. 7(a)-(b)-(c), respectively. As for the lower cavitation velocities investigated before, the material viscosity and the loading time hardly affect the radial stress fields. On the other hand, an important difference with the results reported for $m=0.1$ and $m=0.2$ is that the $\Sigma_{r r} \times \xi$ curves, as they are displayed in this paper with increasing negative values of the radial stress along the $y$-axis, do not show a concave-upward shape. For $m=0.4$, the radial stress decreases almost linearly as we move away from the cavity wall, with a quick drop 


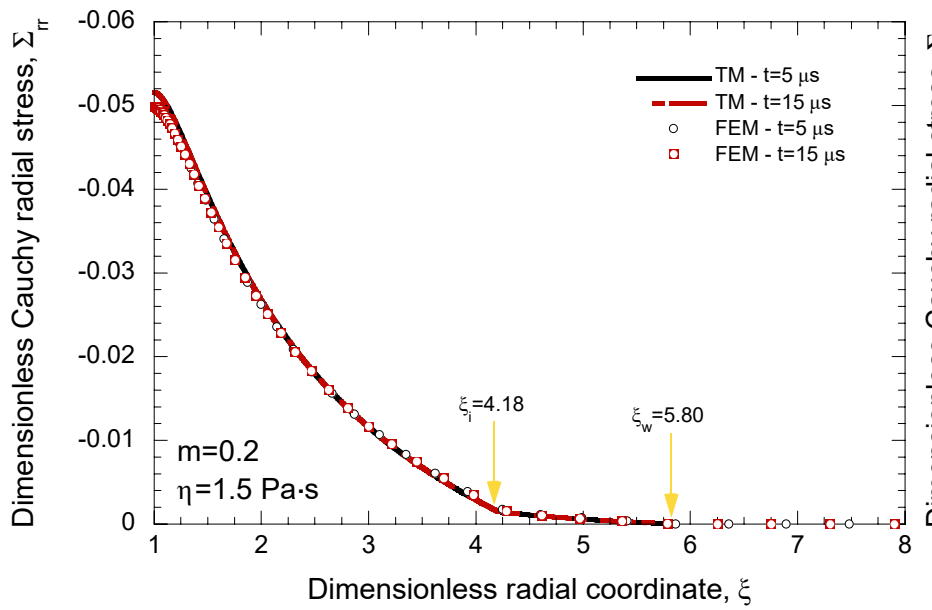

(a)

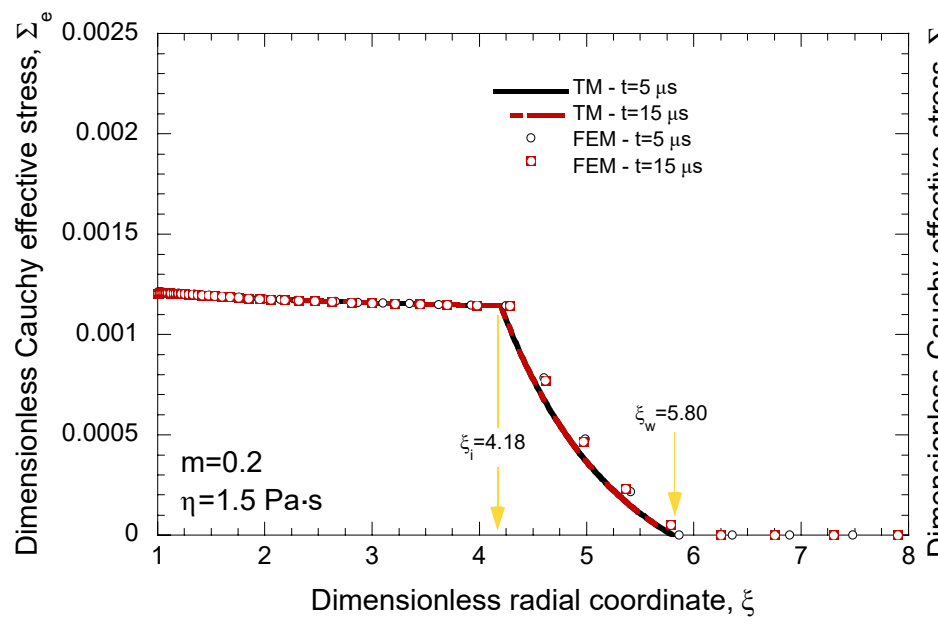

(c)

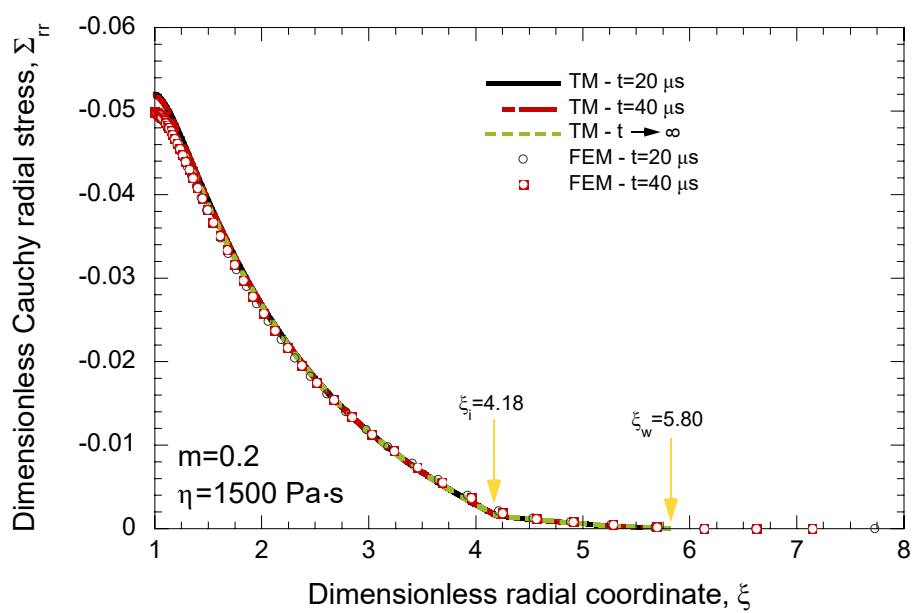

(b)

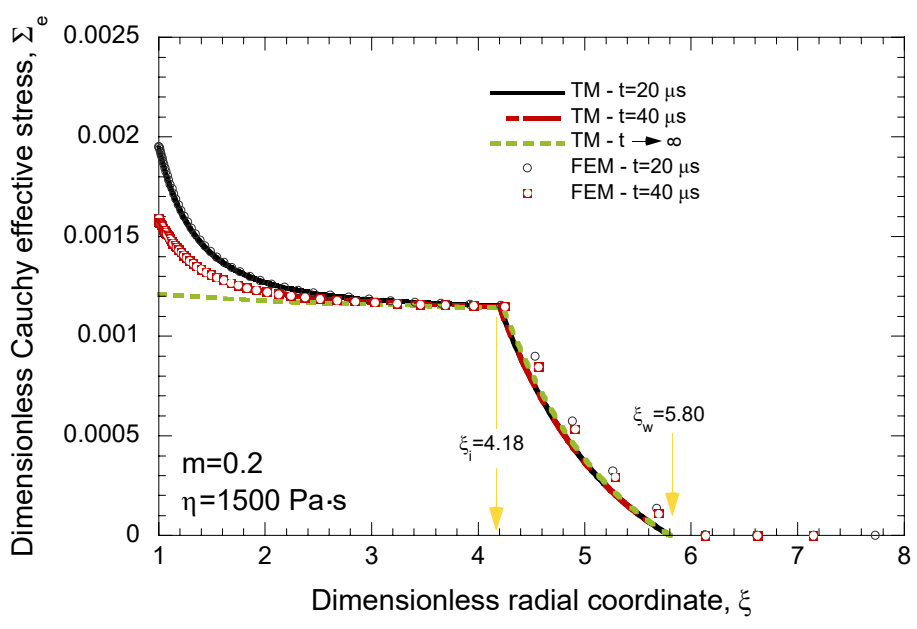

(d)

Figure 6: Comparison between theoretical results (TM) and finite element results (FEM) for $m=0.2$. Dimensionless radial Cauchy stress $\Sigma_{r r}$ versus dimensionless radial coordinate $\xi$ for (a) $\eta=1.5 \mathrm{~Pa} \cdot \mathrm{s}$ and (b) $\eta=1500 \mathrm{~Pa} \cdot \mathrm{s}$. Dimensionless effective Cauchy stress $\Sigma_{e}$ versus dimensionless radial coordinate $\xi$ for $(\mathrm{c}) \eta=1.5 \mathrm{~Pa} \cdot \mathrm{s}$ and $(\mathrm{d}) \eta=1500 \mathrm{~Pa} \cdot \mathrm{s}$. The theoretical results were obtained without artificial viscosity, $c_{0}=0$ and $c_{L}=0$. 
at $\xi_{p}=2.29$ which corresponds to the shock wave. The shock wave location virtually coincides with the elastic / elasto-viscoplastic interface $\xi_{p} \approx \xi_{i}$ (see also refs. $[31,18,3]$ ).

Figures 8(a)-(b)-(c) display the variation of the effective Cauchy stress $\Sigma_{e}$ with the dimensionless radial coordinate $\xi$. For $\eta=1.5 \mathrm{~Pa} \cdot \mathrm{s}$, Fig. 8(a), the effective stress field is hardly dependent on the loading time considered. However, the time dependence of the effective stress fields becomes more important as the viscosity parameter increases. For $\eta=150 \mathrm{~Pa} \cdot \mathrm{s}$, Fig. 8(b), the value of $\Sigma_{e}$ near the cavity is slightly smaller for $t=10 \mu \mathrm{s}$ than for $t=5 \mu \mathrm{s}$. Moreover, for the greatest material viscosity considered $\eta=1500 \mathrm{~Pa} \cdot \mathrm{s}$, Fig. $8(\mathrm{c})$, the value of the effective stress at the cavity wall for $7 \mu \mathrm{s}$ is $\approx 1.75$ times greater than for $20 \mu \mathrm{s}$. Note that, for the latter loading time, near the cavity, the effective stress is approximately 80 times smaller than the absolute value of the radial stress, i.e. the triaxiality is roughly -80 . Note that for high cavitation velocities and applied pressures, the radial, circumferential and azimuthal stresses are similar, and much greater in absolute value than the equivalent stress (see Eq. (12)). Therefore, the ratio between the radial and the effective stress is roughly the stress triaxiality. The large hydrostatic pressure explains that, while the effective stress fields are strongly dependent on the loading time, the radial stress fields are not (compare Figs. 7 and 8). Moreover, the peak of effective stress located $\xi_{p}=2.29$ corresponds to the shock wave. The formation of a shock leads to a local increase of the strain rate [39, 31], which boosts the effective stress. As the loading time increases, the peak of stress becomes smaller (as further discussed below).

Figures 9 and 10 show the radial and effective Cauchy stress fields predicted by the theoretical model and the finite element simulations for the greatest cavitation velocity investigated in this work $m=0.6$. Note that the intensity of the shock increases with the cavitation velocity. The artificial viscosity with $c_{0}=0.55$ and $c_{L}=0.25$ was used to obtain the results for $\eta=1.5$ and $150 \mathrm{~Pa} \cdot \mathrm{s}$ (note that in Figs. 7 and 8 the results for $m=0.4$ and $\eta=150 \mathrm{~Pa} \cdot \mathrm{s}$ were obtained without artificial viscosity). In contrast, in the case of $\eta=1500 \mathrm{~Pa} \cdot \mathrm{s}$, for the loading times considered, the artificial viscosity was not needed to integrate the theoretical model. Nevertheless, for $t>12 \mu \mathrm{s}$ the artificial viscosity becomes also necessary to capture the shock wave for $\eta=1500 \mathrm{~Pa} \cdot \mathrm{s}$. As for the lower cavitation velocities, the agreement between theoretical predictions and finite element calculations is excellent.

Figures 9(a)-(b)-(c) show the radial Cauchy stress $\Sigma_{r r}$ versus the dimensionless radial coordinate $\xi$ for $\eta=1.5$, 150 and $1500 \mathrm{~Pa} \cdot \mathrm{s}$, respectively. The $\Sigma_{r r} \times \xi$ curves, as they are displayed in this paper with increasing negative values of the radial stress along the $y$-axis, feature a concave-downward shape with a drastic drop at $\xi_{p}=1.68$ which corresponds to the shock wave location. Note that, as in the case of $m=0.4$ shown in Figs. 7 and 8 , we have that $\xi_{p} \approx \xi_{i}$. Both in the theoretical model and the finite element simulations, the change in the radial stress induced by the shock, while abrupt, is not a discontinuity, i.e. the shock has a finite width due to the regularizing effect of artificial/material viscosity. In the case of $\eta=1500 \mathrm{~Pa} \cdot \mathrm{s}$, Fig. 9(c), it becomes apparent that the width 


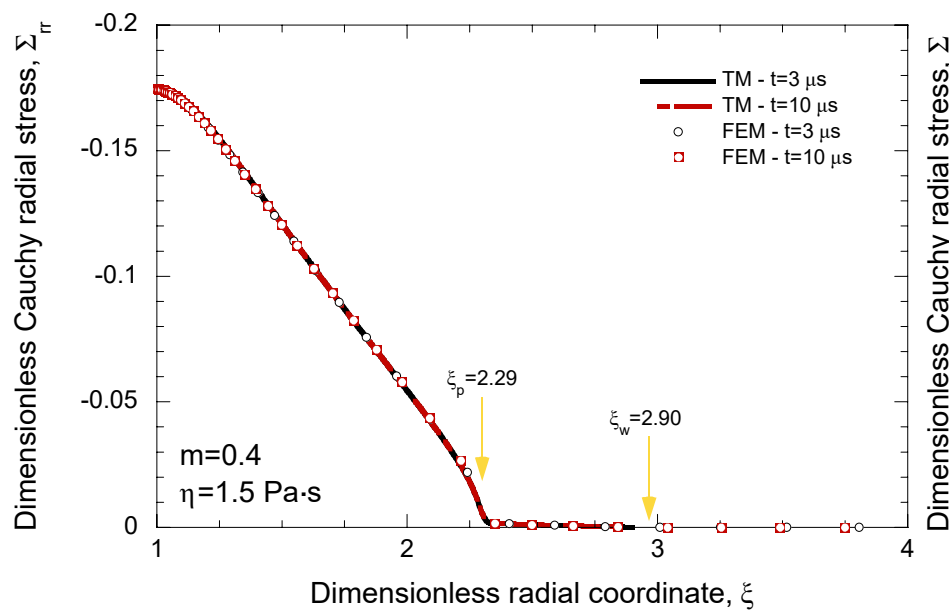

(a)

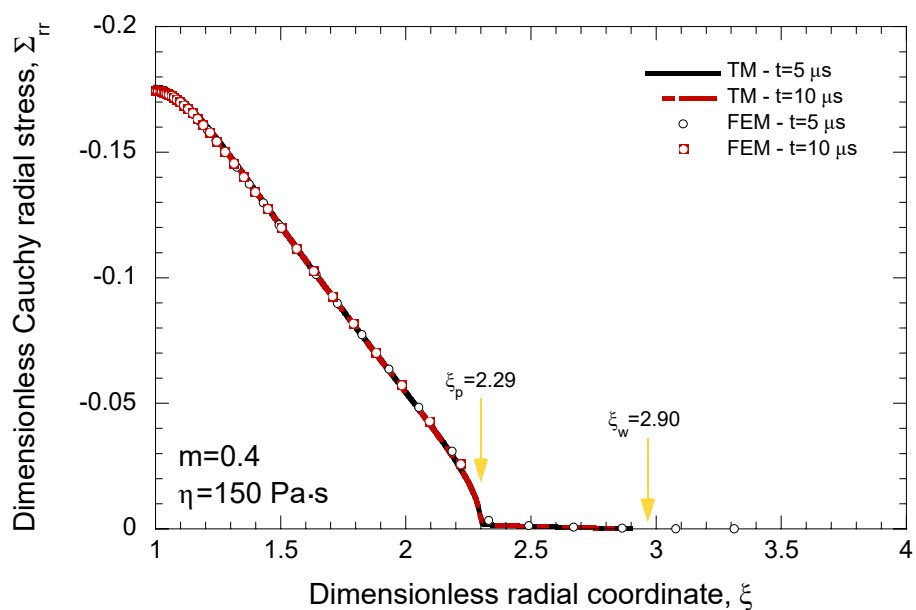

(b)

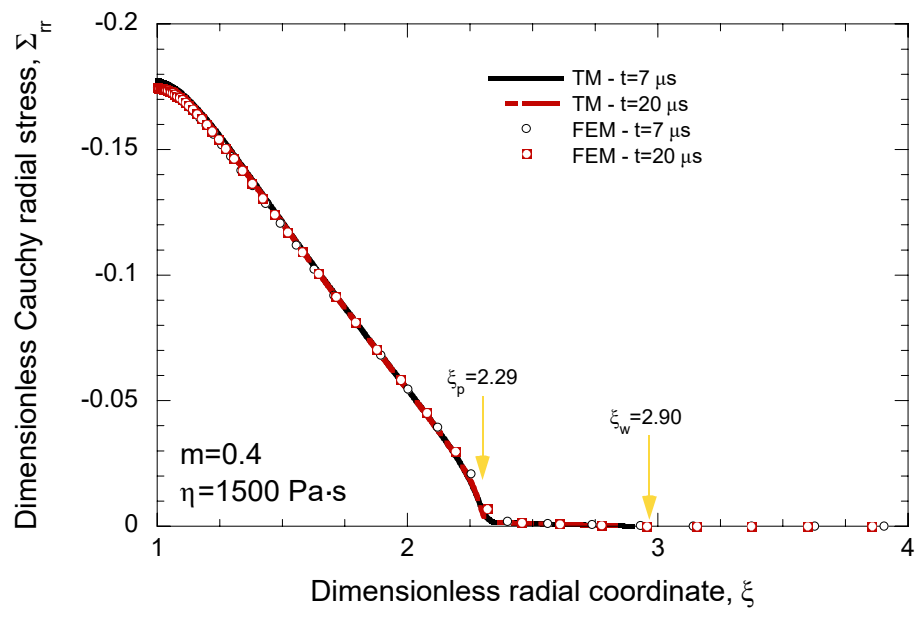

(c)

Figure 7: Comparison between theoretical results (TM) and finite element results (FEM) for $m=0.4$. Dimensionless radial Cauchy stress $\Sigma_{r r}$ versus dimensionless radial coordinate $\xi$ for (a) $\eta=1.5 \mathrm{~Pa} \cdot \mathrm{s}$, (b) $\eta=150 \mathrm{~Pa} \cdot \mathrm{s}$ and $(\mathrm{c}) \eta=1500 \mathrm{~Pa} \cdot \mathrm{s}$. The theoretical results for $\eta=1.5 \mathrm{~Pa} \cdot \mathrm{s}$ were obtained with $c_{0}=0.55$ and $c_{L}=0.25$. In contrast, the theoretical results for $\eta=150 \mathrm{~Pa} \cdot \mathrm{s}$ and $\eta=1500 \mathrm{~Pa} \cdot \mathrm{s}$ were obtained without artificial viscosity, $c_{0}=0$ and $c_{L}=0$. 


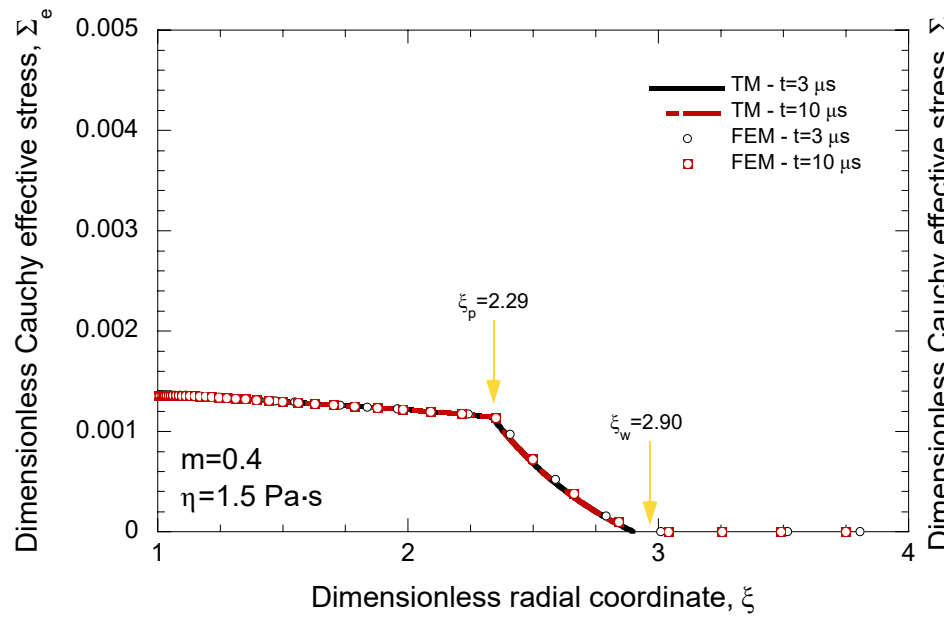

(a)

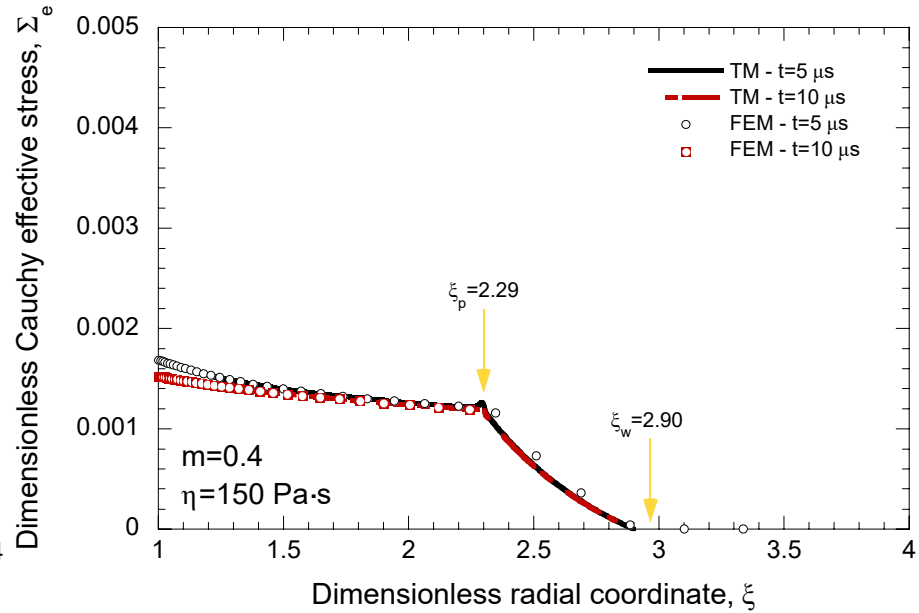

(b)

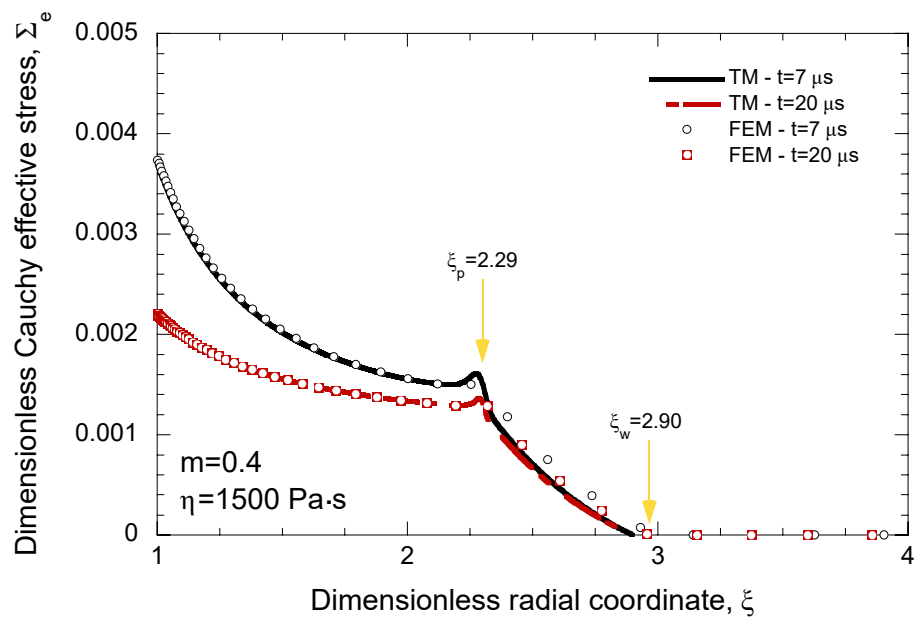

(c)

Figure 8: Comparison between theoretical results (TM) and finite element results (FEM) for $m=0.4$. Dimensionless effective Cauchy stress $\Sigma_{r r}$ versus dimensionless radial coordinate $\xi$ for (a) $\eta=1.5 \mathrm{~Pa} \cdot \mathrm{s}$, (b) $\eta=150 \mathrm{~Pa} \cdot \mathrm{s}$ and $(\mathrm{c}) \eta=1500 \mathrm{~Pa} \cdot \mathrm{s}$. The theoretical results for $\eta=1.5 \mathrm{~Pa} \cdot \mathrm{s}$ were obtained with $c_{0}=0.55$ and $c_{L}=0.25$. In contrast, the theoretical results for $\eta=150 \mathrm{~Pa} \cdot \mathrm{s}$ and $\eta=1500 \mathrm{~Pa} \cdot \mathrm{s}$ were obtained without artificial viscosity, $c_{0}=0$ and $c_{L}=0$. 
of the shock is smaller as the loading times increases, i.e. as the material relaxes, which shows the influence of material viscosity in the shock width. Moreover, the comparison with the results reported in Figs. 7 and 8 shows that the shock wave is located closer to the cavity as $m$ increases (see also refs. $[3,31,18]$ ). Outside the shock front, as for the lower cavitation velocities investigated, the material viscosity hardly affects the radial stress fields, which virtually overlap each other for different loading times and the three viscosity parameters considered (note that the hydrostatic stress is very large).

The evolution of the effective Cauchy stress $\Sigma_{e}$ with the radial coordinate is shown in Figs. 10(a)-(b)-(c). For the smaller material viscosity, Fig. 10(a), the effective stress is largely independent of the loading time. The decrease of $\Sigma_{e}$ with the radial coordinate within the elasto-viscoplastic zone is, mostly, due to the effect of material compressibility on the effective Cauchy stress. The kink in the $\Sigma_{e} \times \xi$ curves corresponds to the elastic / elastoviscoplastic interface $\xi_{i}$, which virtually coincides with the shock wave location (as mentioned before). The decrease of the effective stress for $\xi>\xi_{i}$ corresponds to the elastic zone. For greater values of the viscosity parameter, Figs. 10(b)-(c), the $\Sigma_{e} \times \xi$ curves become time dependent, as in the case of $m=0.4$ shown in Figs. 8(b)-(c). For both $\eta=150 \mathrm{~Pa} \cdot \mathrm{s}$ and $\eta=1500 \mathrm{~Pa} \cdot \mathrm{s}$, within the elasto-viscoplastic zone, the effective stress is greater for $5 \mu \mathrm{s}$ than for $10 \mu$ s, i.e. the material relaxes with the loading time. The drastic increase of effective stress located at $\xi_{p}=1.68$ corresponds to the shock wave. Both finite element simulations and theoretical predictions show that the width of the shock wave and the maximum effective stress attained within the shock wave decrease with the loading time. This result is consistent with the conclusions obtained in ref. [24], where it was shown that viscosity plays a fundamental role in the shock structure.

\section{Concluding remarks and perspectives}

The main contribution of this paper is to extend the dynamic spherical cavity expansion theory developed in refs. [1, 2, 3] to viscoplastic Perzyna-type materials. The theoretical model has been compared with finite element simulations for a wide range of cavitation velocities. We have shown that the material viscosity: (i) increases the time needed for the cavity to reach the constant-velocity expansion, (ii) leads to time-dependent cavitation fields and (iii) facilitates to model the shock waves that emerge at high cavitation velocities. Moreover, the rate-dependent cavity expansion model presented in this work could be readily extended to include, for instance, plastic anisotropy [18], porosity [16, 31], strain hardening [2] and thermal softening [40]. The application of the model to study the influence of material viscosity in the penetration resistance of rate-dependent materials is also left as future work. In this regard, note that the application of constant-velocity cavitation fields to model high-velocity penetration phenomena requires the transient behavior that precedes cavitation to be short which, attending to the results presented in this paper, may not be the case if the viscosity of the material is sufficiently high. 


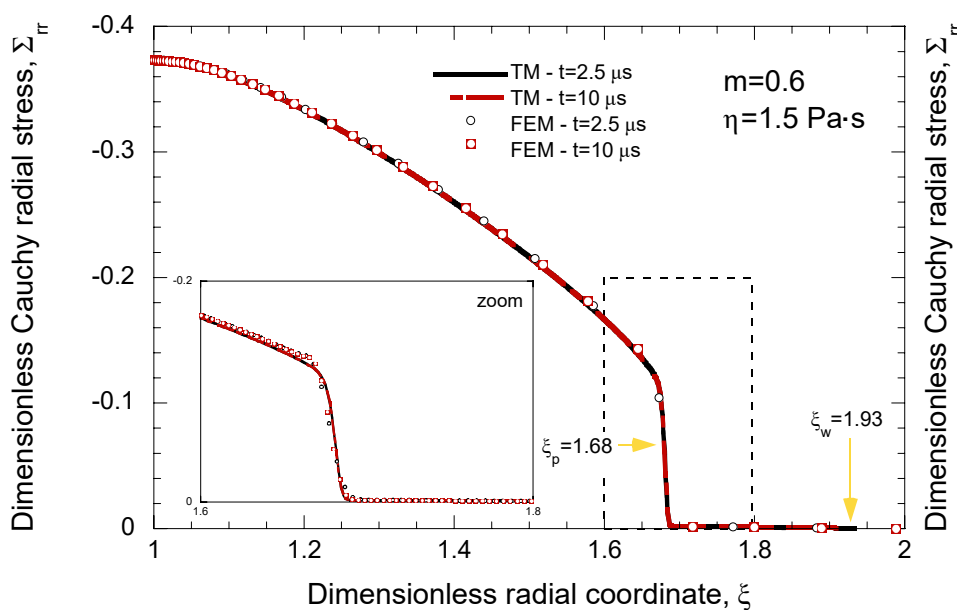

(a)

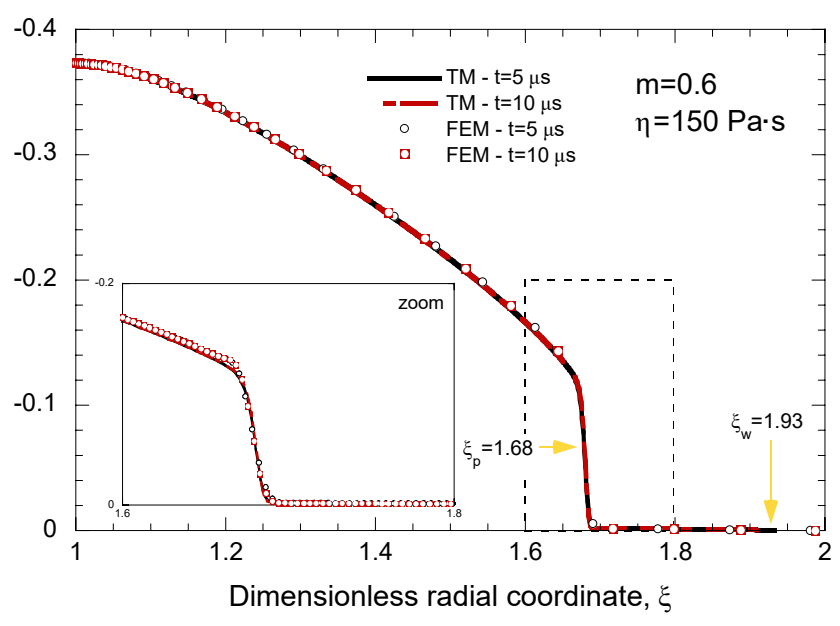

(b)

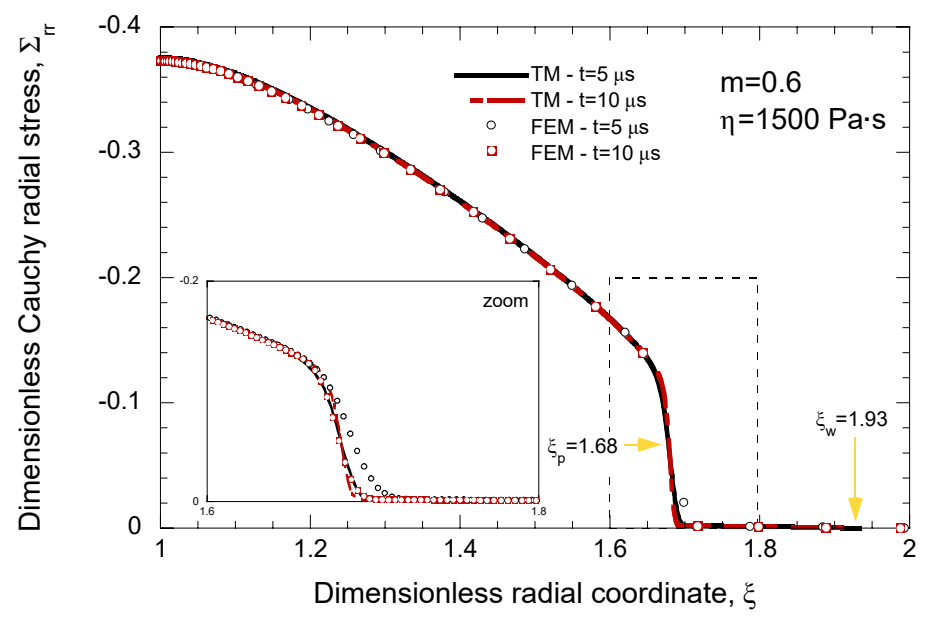

(c)

Figure 9: Comparison between theoretical results (TM) and finite element results (FEM) for $m=0.4$. Dimensionless radial Cauchy stress $\Sigma_{r r}$ versus dimensionless radial coordinate $\xi$ for (a) $\eta=1.5 \mathrm{~Pa} \cdot \mathrm{s}$, (b) $\eta=150 \mathrm{~Pa} \cdot \mathrm{s}$ and $(\mathrm{c}) \eta=1500 \mathrm{~Pa} \cdot \mathrm{s}$. The theoretical results for $\eta=1.5 \mathrm{~Pa} \cdot \mathrm{s}$ and $\eta=150 \mathrm{~Pa} \cdot \mathrm{s}$ were obtained with $c_{0}=0.55$ and $c_{L}=0.25$. In contrast, the theoretical results for $\eta=1500 \mathrm{~Pa} \cdot \mathrm{s}$ were obtained without artificial viscosity, $c_{0}=0$ and $c_{L}=0$. 


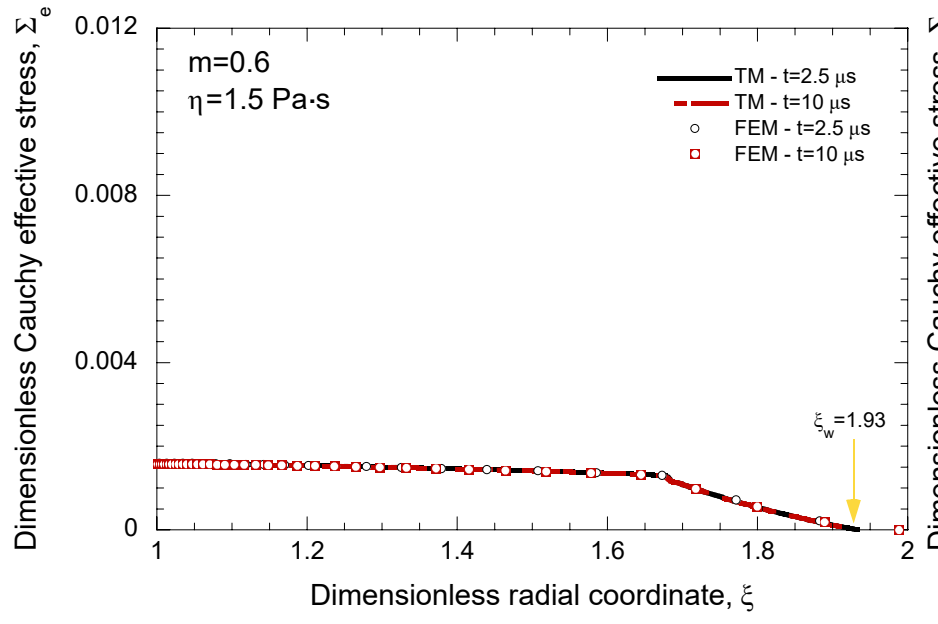

(a)

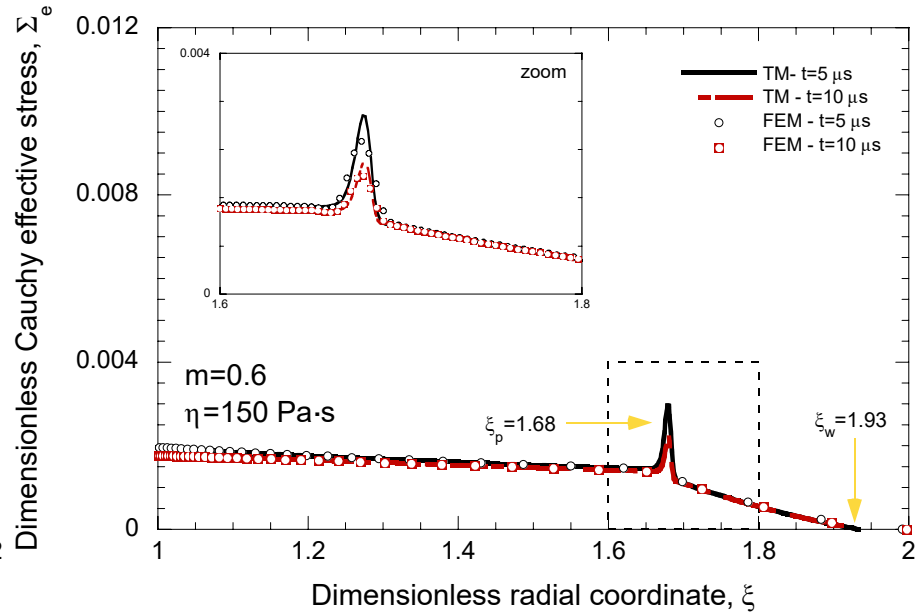

(b)

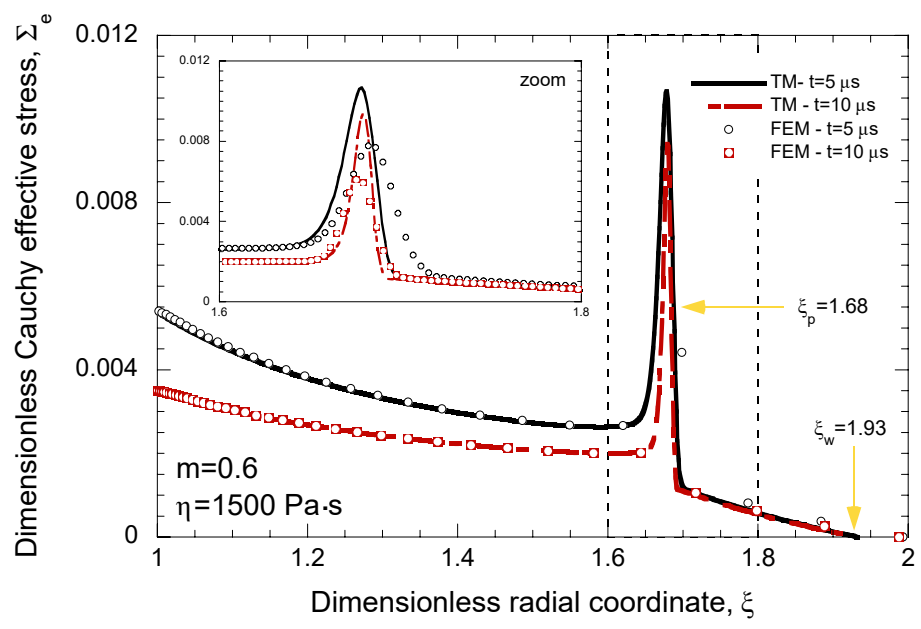

(c)

Figure 10: Comparison between theoretical results (TM) and finite element results (FEM) for $m=0.4$. Dimensionless effective Cauchy stress $\Sigma_{r r}$ versus dimensionless radial coordinate $\xi$ for (a) $\eta=1.5 \mathrm{~Pa} \cdot \mathrm{s}$, (b) $\eta=150 \mathrm{~Pa} \cdot \mathrm{s}$ and $(\mathrm{c}) \eta=1500 \mathrm{~Pa} \cdot \mathrm{s}$. The theoretical results for $\eta=1.5 \mathrm{~Pa} \cdot \mathrm{s}$ and $\eta=150 \mathrm{~Pa} \cdot \mathrm{s}$ were obtained with $c_{0}=0.55$ and $c_{L}=0.25$. In contrast, the theoretical results for $\eta=1500 \mathrm{~Pa} \cdot \mathrm{s}$ were obtained without artificial viscosity, $c_{0}=0$ and $c_{L}=0$. 


\section{Acknowledgements}

The research leading to these results has received funding from the European Research Council (ERC) under the European Union's Horizon 2020 research and innovation programme. Project PURPOSE, grant agreement 758056 .

RR wishes to acknowledge the support of CNPq, Conselho Nacional de Desenvolvimento Científico e Tecnológico, grant number 306058/2018-9.

\section{References}

[1] Durban, D., Masri, R.. Dynamic spherical cavity expansion in a pressure sensitive elastoplastic medium. International Journal of Solids and Structures 2004;41(20):5697-5716.

[2] Masri, R., Durban, D.. Dynamic spherical cavity expansion in an elastoplastic compressible Mises solid. Journal of Applied Mechanics 2005;72(6):887-898.

[3] Cohen, T., Masri, R., Durban, D.. Shock waves in dynamic cavity expansion. Journal of Applied Mechanics 2010;77(4):041009.

[4] Perzyna, P.. The constitutive equations for rate sensitive plastic materials. Quarterly of Applied Mathematics 1963;20:321-332.

[5] Perzyna, P.. Fundamental problems in viscoplasticity. Advances in Applied Mechanics 1966;9:243-377.

[6] ABAQUS/Explicit, . Abaqus Explicit v6.13 User's Manual. Richmond, USA: ABAQUS Inc.; version 6.13 ed.; 2013.

[7] Hopkins, H.. Dynamic expansion of spherical cavities in metals. Progress in Solid Mechanics 1960;1(3):5-16.

[8] Goodier, J.. On the mechanics of indentation and cratering in solid targets of strain-hardening metal by impact of hard and soft spheres. In: Proceedings of the 7th Symposium on Hypervelocity Impact; vol. 3. 1965, p. $215-259$.

[9] Forrestal, M., Luk, V., Brar, N.. Perforation of aluminum armor plates with conical-nose projectiles. Mechanics of Materials 1990;10(1):97 - 105.

[10] Forrestal, M., Brar, N., Luk, V.. Penetration of strain-hardening targets with rigid spherical-nose rods. Journal of Applied Mechanics 1991;58(1):7-10.

[11] Forrestal, M.J., Warren, T.L.. Perforation equations for conical and ogival nose rigid projectiles into aluminum target plates. International Journal of Impact Engineering 2009;36(2):220-225. 
[12] Masri, R., Durban, D.. Deep penetration analysis with dynamic cylindrical cavitation fields. International Journal of Impact Engineering 2009;36(6):830 - 841.

[13] Cohen, T., Masri, R., Durban, D.. Ballistic limit predictions with quasi-static cavitation fields. International Journal of Protective Structures 2010;1:235-255.

[14] Durban, D., Fleck, N.A.. Spherical cavity expansion in a Drucker-Prager solid. Journal of Applied Mechanics 1997;64:743-750.

[15] Fleck, N., Otoyo, H., Needleman, A.. Indentation of porous solids. International Journal of Solids and Structures 1992;29(13):1613-1636.

[16] Cohen, T., Durban, D.. Hypervelocity cavity expansion in porous elastoplastic solids. Journal of Applied Mechanics 2013;80(1):011017.

[17] Cohen, T., Durban, D.. Fundamental solutions of cavitation in porous solids: a comparative study. Acta Mechanica 2013;224(8):1695-1707.

[18] dos Santos, T., Vaz-Romero, A., Rodríguez-Martínez, J.A.. Dynamic cylindrical cavity expansion in orthotropic porous ductile materials. International Journal of Impact Engineering 2019;132:103325.

[19] Warren, T.L., Forrestal, M.J.. Effects of strain hardening and strain-rate sensitivity on the penetration of aluminum targets with spherical-nosed rods. International Journal of Solids and Structures 1998;35(28):3737 $-3753$.

[20] Cohen, T., Durban, D.. Steady shock waves in porous plastic solids. International Journal of Solids and Structures 2015;71:70 - 78 .

[21] Cleja-Tigoiu, S., Cazacu, O., Tigoiu, V.. Dynamic expansion of a spherical cavity within a rate-dependent compressible porous material. International Journal of Plasticity 2008;24(5):775 - 803.

[22] Buchely, M.F., Marañon, A.. An engineering model for the penetration of a rigid-rod into a Cowper-Symonds low-strength material. Acta Mechanica 2015;226(9):2999-3010.

[23] Buchely, M.F., Marañon, A.. Study of steady cavitation assumptions in strain-rate-sensitive solids for rigid projectile penetrations. Acta Mechanica 2016;227(10):2969-2983.

[24] Molinari, A., Ravichandran, G.. Fundamental structure of steady plastic shock waves in metals. Journal of Applied Physics 2004;95(4):1718-1732.

[25] dos Santos, T., Ramos, G.R., Rossi, R.. A note on overstress and over-thermodynamic forces derivation for elasto-viscoplastic media: Thermodynamic analysis of an isothermal relaxation process. International Journal of Engineering Science 2015;93:13-30. 
[26] Holzapfel, G.. Nonlinear Solid Mechanics: A Continuum Approach for Engineering. Richmond, USA: Wiley; 2000. ISBN 978-0-471-82319-3.

[27] Kanninen, M.F., Mukherjee, A.K., Rosenfield, A.R., Hahn, G.T.. The Speed of Ductile-Crack Propagation and the Dynamics of Flow in Metals. Berlin, Heidelberg: Springer Berlin Heidelberg; 1968, p. 96-133.

[28] Perzyna, P.. Adiabatic shear band localization fracture of solids in dynamic loading processes. Journal de Physique IV Colloque 1994;04(C8):C8-441-C8-446.

[29] Glema, A., Łodygowski, T., Sumelka, W., Perzyna, P.. The numerical analysis of the intrinsic anisotropic microdamage evolution in elasto-viscoplastic solids. International Journal of Damage Mechanics 2009;18(3):205231.

[30] Perzyna, P.. Application of the Thermodynamical Theory of Elasto-Viscoplasticity in Modern Manufacturing Processes. Vienna: Springer Vienna. ISBN 978-3-7091-0427-9; 2011, p. 227-376.

[31] dos Santos, T., N'souglo, K.E., Rodríguez-Martínez, J.A.. Dynamic spherical cavity expansion in Gurson materials with uniform and non-uniform distributions of porosity. Mechanics of Materials 2019;134:115-131.

[32] Lew, A., Radovitzky, R., Ortiz, M.. An artificial-viscosity method for the lagrangian analysis of shocks in solids with strength on unstructured, arbitrary-order tetrahedral meshes. Journal of Computer-Aided Materials Design 2001;8(2):213-231.

[33] Wilkins, M.L.. Use of artificial viscosity in multidimensional fluid dynamic calculations. Journal of Computational Physics 1980;36(3):281 - 303.

[34] Rodríguez-Martínez, J.A., Cohen, T., Zaera, R.. Approaching steady cavitation: The time scale in hypervelocity cavity expansion in work hardening and transformation hardening solids. International Journal of Impact Engineering 2014;73:43 - 55.

[35] Aranda-Iglesias, D., Vadillo, G., Rodríguez-Martínez, J.A.. Oscillatory behaviour of compressible hyperelastic shells subjected to dynamic inflation: A numerical study. Acta Mechanica 2017;228(6):2187-2205.

[36] Alfano, G., Angelis, F.D., Rosati, L.. General solution procedures in elasto/viscoplasticity. Computer Methods in Applied Mechanics and Engineering 2001;190(39):5123 - 5147.

[37] Careglio, C., Canales, C., García Garino, C., Mirasso, A., Ponthot, J.P.. A numerical study of hypoelastic and hyperelastic large strain viscoplastic Perzyna type models. Acta Mechanica 2016;227(11):3177-3190.

[38] Cohen, T., Molinari, A.. Dynamic cavitation and relaxation in incompressible nonlinear viscoelastic solids. International Journal of Solids and Structures 2015;69-70:544 - 552. 
[39] Czarnota, C., Molinari, A., Mercier, S.. The structure of steady shock waves in porous metals. Journal of the Mechanics and Physics of Solids 2017;107:204 - 228.

[40] Masri, R.. The effect of adiabatic thermal softening on specific cavitation energy and ductile plate perforation. International Journal of Impact Engineering 2014;68:15-27. 